\title{
Zur Bedeutung von Sprache im Fachunterricht
}

Nachfolgend werden basierend auf einer begrifflichen Abgrenzung von Alltags-, Bildungs- und Fachsprache (vgl. Abschn. 2.1) die verschiedenen Rollen von Sprache im Geographieunterricht diskutiert (vgl. Abschn. 2.2). Dieses Kapitel soll offenlegen, warum sprachsensibler Geographieunterricht eine sinnvolle und notwendige Ausrichtung des Geographieunterrichts ist. Mit diesem Wissen werden anschließend verschiedene Studien zum Zusammenhang von Sprache und Fachkompetenz sowie relevante Interventionsstudien aus weiteren Fächern vorgestellt (vgl. Abschn. 2.3).

\subsection{Sprache ist nicht gleich Sprache: eine definitorische Abgrenzung}

Deutsch, das ist eine von circa 6.500 bis 8.000 Einzelsprachen $^{1}$ (vgl. Vogel 2012, S. 1; Wiater 2006, S. 57), das vielfältige Varietäten aufweist. Diese sind mitunter von der geographischen Lage und der sozialen Zugehörigkeit der Sprecher*innen, aber auch von der Funktion einer sprachlichen Äußerung sowie der Situation, in der sie getätigt wird, abhängig. Eine Einzelsprache ist also mannigfaltig; und zwar auf eine Weise, die es nahelegt, dass auch sprachliche Kompetenz nicht eindimensional sein kann. Was unter sprachlicher Kompetenz gefasst wird und was nicht, welche Eigenschaften eine Person zu einem*r sprachlich kompetenten Sprecher*in machen, sind Definitionsfragen. So werden Sprachen und

\footnotetext{
${ }^{1}$ Angaben zur Anzahl der Sprachen auf der Welt variieren stark. Eine allseits akzeptierte Klassifikation von Sprache ist bisher noch nicht geleistet (vgl. Videosott 2006, S. 52).
} 
deren Varietäten von Autor*in zu Autor*in unterschiedlich definiert und voneinander abgegrenzt. Die verschiedenen Konstrukte und Definitionen existieren völlig inkonsistent parallel nebeneinander.

Die Theorie der Einzelsprache als Sammlung vieler Varietäten geht nicht nur davon aus, dass es eine Norm als solche nicht gibt, sondern behauptet auch, dass das, was allgemein als Hoch- oder Standarddeutsch bezeichnet wird, eine Varietät des Deutschen ist (vgl. Becker/Hundt 1998, S. 118). Für den Begriff Varietät finden sich in der Literatur noch eine Reihe anderer Begriffe, die weitgehend synonym verwendet werden: Subsprache, Jargon, Genre, Stil, Register und Lekt (vgl. u. a. Adamzik 1998; Feilke 2012a; Hess-Lüttich 1998; Hoffmann 1998; Höttecke et al. 2017; Roelcke 2020). Um die verschiedenen Ausprägungen des Deutschen zu bezeichnen, wird nachfolgend der Begriff Varietät gewählt, der als „eine Menge von Varianten, die in Bezug auf Variablen fixiert sind“ (Becker/Hundt 1998, S. 119) definiert ist. Durch die Bestimmung der Variablen ist also eine Klassifikation in verschiedene sprachliche Varietäten möglich. Dabei ist die Variation die Veränderung selbst und die Varietät das Ergebnis der Variation. Man unterscheidet bei synchroner ${ }^{2}$ Sprachbetrachtung drei verschiedene Dimensionen der Varietäten: diatopische, diastratische und diaphasische Dimensionen (vgl. Abb. 2.1).

Regionale Varietäten (diatopische Dimension) sind vor allem in Form von Dialekten bekannt. Soziale Varietäten (diastratische Dimension) hingegen betreffen eine bestimmte soziale Schicht und werden oft als Soziolekte bezeichnet. So sprechen Jugendliche mit ihren Peers in der Regel anders als mit ihren Lehrer*innen. Für die vorliegende Arbeit von besonderer Relevanz ist die Kategorie der funktional-situativen Varietäten (diaphasische Dimension). Synonym zu diesem Begriff wird in der Fachliteratur häufig von Registern gesprochen. Eine trennscharfe Unterscheidung insbesondere zwischen den diaphasischen und diastratischen Varietäten ist allerdings nicht immer möglich (vgl. Michalak 2012b, S. 69). Ein denkbarer Zugang, um die Kriterien für ein Register zu definieren, gründet auf der systematisch-funktionalen Linguistik mit ihrem prominentesten Vertreter Halliday. Ihm zufolge sind Register ,a set of meanings that appropriate to a particular function of language, together with words and structures which express these meanings" (Halliday 1978, S. 195). Mittels der Kriterien field (Bezugsbereich), mode (Medium/Konzeption) und tenor (Interaktionssituation) lassen sich dieser Logik zufolge verschiedene sprachliche Register unterscheiden. Für die folgenden Begriffsabgrenzungen sind die Kriterien der funktionalen

${ }^{2}$ Bei diachroner Betrachtung spielen als vierte Varietät auch historische Varietäten eine Rolle. 


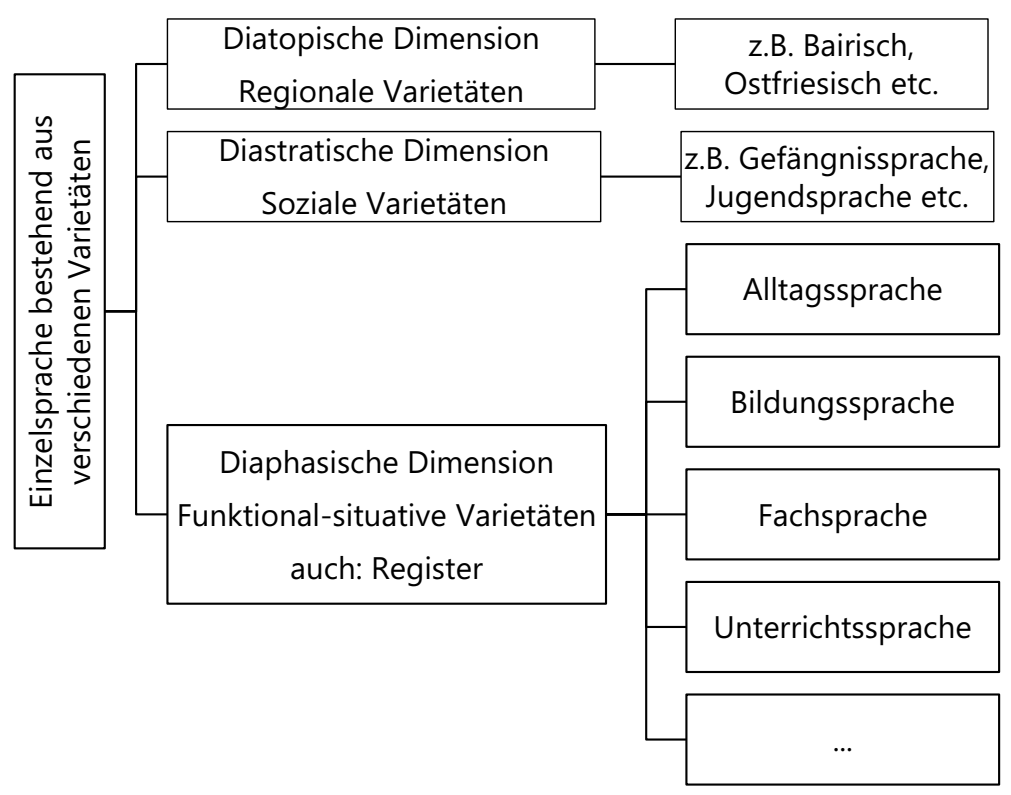

Abb. 2.1 Synchrone Sprachvarietäten im Überblick. (Eigene Darstellung)

Grammatik, die den zweckgebundenen Charakter von Sprache fokussieren, maßgeblich (vgl. Dik/Hengeveld 1997; Halliday 1978; Hengeveld/Mackenzie 2008; Leckie-Tarry 1995). Sie werden angereichert durch Zugänge von Bernstein (1971) aus der Soziolinguistik, der Linguistik mit Koch/Oesterreicher (1985) sowie der Erziehungswissenschaft mit Cummins, Jim (1979), um ein umfassendes Bild der sprachlichen Register zu zeichnen.

Das Kriterium field bezeichnet ,den Redegegenstand, den Inhalt der Verständigung, das Thema eines Textes [...], den Texttyp, [...] das Sach-, Fachund Arbeitsgebiet, in dem sprachlich gehandelt wird [und] über das sprachlich verhandelt wir" (Hess-Lüttich 1998, S. 210). Mode unterscheidet zwischen dem Medium und der Konzeption einer Sprachäußerung (vgl. Koch/Oesterreicher 1985, S. 17). Es wird einerseits darin unterschieden, ob eine Äußerung gesagt (medial mündlich) oder geschrieben (medial schriftlich) wird und andererseits, ob diese Äußerung schriftlich (eher planvoll) oder mündlich (eher spontan) konzipiert wurde (vgl. Tab. 2.1). 
Tab. 2.1 Unterscheidung sprachlicher Äußerungen nach Medium und Konzeption mit Beispielen

\begin{tabular}{l|l|l}
\hline & konzeptionell mündlich & konzeptionell schriftlich \\
\hline $\begin{array}{l}\text { medial } \\
\text { mündlich }\end{array}$ & $\begin{array}{l}\text { Gespräch mit Freund*innen } \\
\text { Gespräch mit Mitschüler*innen in der Pause }\end{array}$ & $\begin{array}{l}\text { Politische Rede } \\
\text { Wissenschaftlicher Vortrag } \\
\text { Referat in der Schule }\end{array}$ \\
\hline $\begin{array}{l}\text { medial } \\
\text { schriftlich }\end{array}$ & $\begin{array}{l}\text { Grußkarte } \\
\text { SMS }\end{array}$ & $\begin{array}{l}\text { Gesetzestext } \\
\text { Fachliteratur }\end{array}$ \\
\hline
\end{tabular}

Quelle: verändert nach Koch/Oesterreicher 1994, S. 588

Zuletzt wird ein sprachliches Register über den tenor definiert, das heißt über das Verhältnis der Kommunikationspartner*innen zueinander (vgl. Riebling 2013, S. 113). Handelt es sich beispielsweise um ein freundschaftliches, privates Verhältnis oder ein professionelles Verhältnis unter Fachkolleg*innen? Trotz dieser Kriterien ist eine trennscharfe Unterscheidung von Registern nicht immer möglich.

\subsubsection{Alltagssprache vs. Bildungssprache}

Unter Berücksichtigung der drei genannten Kriterien werden nachfolgend Alltags- und Bildungssprache voneinander abgegrenzt und definiert. Abgesehen von der im Folgenden dargestellten funktionalen Unterscheidung zwischen field, mode und tenor werden Überlegungen des Soziologen Bernstein (1971) als wichtige Grundlage für die Begriffsabgrenzung von Bildungs- und Alltagssprache integriert. Er unterscheidet bereits in den frühen 1970er-Jahren eine public language, der sich vorwiegend die Arbeiterklasse bedient von einer formal language, die ihm zufolge den höheren sozialen Schichten vorbehalten ist. Bemerkenswert daran ist, dass Bernstein (1971) in diesen Überlegungen bereits ein soziales Gefälle in der Verwendung von Alltags- und Bildungssprache sieht, das lange Jahre im Schulsystem kaum Beachtung fand. Erst in den letzten 20 Jahren wird diese Schieflage aufgrund der Ergebnisse verschiedener Schulleistungsstudien bedeutsam.

\section{Bezugsbereich (field)}

In alltagssprachlichen Situationen ist es möglich, dass Sätze unvollständig oder ungrammatisch sind und dennoch von den Gesprächspartner*innen verstanden und akzeptiert werden, zum Beispiel: 

A: „Wie komme ich zur Schule?“
B: „Geradeaus, links.“

Die Kommunikation gelingt, weil der Erlebniskontext des Gesagten bei den Kommunizierenden derselbe ist (vgl. Lange/Gogolin 2010, S. 12). Die Bedeutung dessen, was gesagt wird, geht dabei meist nicht (nur) aus der verbalen Sprache, sondern (auch) aus nonverbalen und paraverbalen Signalen, wie Gestik, Mimik, Satzmelodie oder Betonung hervor (vgl. Schmölzer-Eibinger et al. 2013, S. 17). Bei der Wegbeschreibung zur Schule, die möglicherweise noch durch Zeigebewegungen unterstützt wird, ist es überflüssig, genaue Angaben zu Strecke und Himmelsrichtung des Weges zu machen, da sich diese aus dem unmittelbaren, gemeinsamen Erlebniskontext erschließen. Der Fokus liegt auf der Verständigung von gemeinsamen Inhalten, nicht auf sprachlicher Korrektheit. Insbesondere in informellen Kontexten, wie zum Beispiel in der Pause, auf dem Schulweg oder in der Freizeit, wird von Alltagssprache ${ }^{3}$ Gebrauch gemacht. Wenn in Alltagssprache gesprochen oder geschrieben wird, so betrifft das den Bezugsbereich des Alltags wie Personen, Tätigkeiten oder Ereignisse (vgl. Riebling 2013, S. 115). Schüler*innen dient Alltagssprache außerhalb der Schule zur Bewältigung ihres Alltags; sie machen sich mit ihr verständlich und drücken Gefühle und Wünsche aus. Alltagssprache ist dementsprechend gekennzeichnet durch Emotionalität, subjektive Bewertungen und ausdrucksstarke, bildreiche Begriffe (vgl. Michalak et al. 2015, S. 48). Im Rahmen der Bildungssprache ist dies eher nicht der Fall; Objekte wie zum Beispiel Stadtpläne, Gesetze, Nachrichten (auch über Personen) oder Anträge bilden den Gesprächsanlass. Bildungssprache ist in verschiedenen (Bildungs-)Kontexten von Bedeutung, die ,in konzeptionelle und explizite Zusammenhänge eingebunden“ (Riebling 2013, S. 116) sind. Neben Schule und Ausbildung sind damit auch all jene Kommunikationssituationen gemeint, in denen ein formeller Sprachgebrauch gefordert wird, wie zum Beispiel bei Behörden, Ärzt*innenbesuchen oder im beruflichen Umfeld (vgl. Kurtz et al. 2015, S. 2).

Die Bildungssprache ist die Sprache, die überwiegend in den Massenmedien, in Fernsehen, Rundfunk, Tages- und Wochenzeitungen benutzt wird. Sie unterscheidet sich von der Umgangssprache durch die Disziplin des schriftlichen Ausdrucks und durch

\footnotetext{
${ }^{3}$ Synonym zum Begriff der Alltagssprache werden in der Literatur auch „Umgangssprache“ (vgl. Habermas 1981a) oder „Allgemeinsprache“ (Ahrenholz 2010; Michalak/Bachtsevanidis 2012) verwendet. Im Rahmen dieser Arbeit wird nur der Begriff „Alltagssprache“ verwendet.
} 
einen differenzierteren, Fachliches einbeziehenden Wortschatz; andererseits unterscheidet sie sich von Fachsprachen dadurch, daß sie grundsätzlich für alle offensteht, die sich mit den Mitteln der allgemeinen Schulbildung ein Orientierungswissen verschaffen können. (vgl. Habermas 1981b, S. 330)

\section{Medium/Konzeption (mode)}

Alltagssprache ist durch konzeptionelle Mündlichkeit geprägt, orientiert sich also an der mündlichen Kommunikationsstruktur, was häufig mit geringerer Explizitheit einhergeht (vgl. Höttecke et al. 2017, S. 2). „Die Unmittelbarkeit der ,gesprochenen ' Kommunikation [...] ermöglicht eine größere Spontaneität; die Planung kann weniger aufwendig - sozusagen während des Äußerungsaktes selber - erfolgen“ (Koch/Oesterreicher 1985, S. 20). Bildungssprache im Gegensatz dazu ist durch konzeptionelle Schriftlichkeit geprägt; entspricht folglich den Merkmalen der Schriftsprache, wird aber auch medial mündlich verwendet (vgl. Habermas 1981b, S. 330).

\section{Interaktionssituation (tenor)}

Die Interaktionssituation wird durch das soziale Verhältnis der Sprecher*innen zueinander beschrieben. In formellen Kontexten ist das Verhältnis zum Gegenüber anders als in informellen, privaten Alltagssituationen. Insbesondere im öffentlichen Rahmen werden soziale Rollen vorgegeben, die das Sprechen beeinflussen. Selbstverständlich wird auch die alltägliche Lebenswelt in Rollen strukturiert, „sie ist aber insgesamt durch eine stärkere Personenorientierung gekennzeichnet als der institutionelle Kontext" (Riebling 2013, S. 122). Alltagssprache zeichnet sich tendenziell durch eine größere emotionale Nähe zum*r Kommunikationspartner*in aus (vgl. Koch/Oesterreicher 1994, S. 588), wohingegen in der Bildungssprache ein unpersönlicher tenor vorherrscht (vgl. Ortner 2009, S. 2228). Im bildungssprachlichen, so auch im schulischen Kontext überwiegen asymmetrische Interaktionsbeziehungen, die sich darin ausdrücken, dass ,den interagierenden Personen in Abhängigkeit von ihren Status bestimmte Spektren an kommunikativen Handlungsmöglichkeiten eröffnet [werden; eigene Ergänzung]“ (Riebling 2013, S. 123). Im Geographieunterricht sind diese Asymmetrie sowie das Wissensgefälle und die weiteren Aspekte der Bezugssituationen im Verhältnis zwischen Lehrperson und Schüler*in ebenfalls abgebildet.

Im Gegensatz zu Alltagssprache zeichnet sich Bildungssprache also eher durch Distanz denn durch Nähe aus; Koch/Oesterreicher (1985) sprechen in diesem Kontext auch von „Sprache der Nähe“" und „Sprache der Distanz“: 
Die Kombination ,Dialog', , freier Sprecherwechsel', ,Vertrautheit der Partner', ,faceto-face-Interaktion', ,freie Themenentwicklung', ,keine Öffentlichkeit', ,Spontaneität', ,starkes Beteiligtsein', ,Situationsverschränkung', etc. charakterisiert den Pol ,gesprochen'. Die ihm entsprechende Kommunikationsform läßt sich am besten auf den Begriff Sprache der Nähe bringen. Analog charakterisiert die Kombination von ,Monolog', ,kein Sprecherwechsel', ,Fremdheit der Partner', ,räumliche und zeitliche Trennung', ,festes Thema', , völlige Öffentlichkeit', ,Reflektiertheit', ,geringes Beteiligtsein', ,Situationsentbindung', etc. den Pol ,geschrieben'. Die ihm entsprechende Kommunikationsform definieren wir als Sprache der Distanz. (Koch/Oesterreicher 1985, S. 21)

Ist der Erlebniskontext der Sprecher*innen nicht derselbe, so liegt eine gröBere Distanz zwischen Gesagtem und eigentlich Gemeintem vor; zwischen Bezeichnendem und Bezeichnetem (vgl. Gogolin/Lange 2011, S. 112; Saussure 1913). „Um diese Diskrepanz zu überwinden, sind sprachlich komplexe Strukturen notwendig, deren Form sich [...] an dem Regelwerk orientiert, das für die geschriebene Sprache gilt" (Lange/Gogolin 2010, S. 12). Das weiter oben genannte Beispiel der alltagssprachlichen Wegbeschreibung illustriert dies gut (vgl. S. 24). Während die Wegbeschreibung aufgrund des gemeinsamen Erlebniskontexts auch ohne komplexe sprachliche Strukturen gut verständlich ist, wären die gleichen Aussagen ohne Kontext nicht mehr verständlich. Der Zusammenhang zwischen sprachlich einfach/komplex und Verständlichkeit ist allerdings nicht immer gegeben. Mitunter sind verkürzte Strukturen schwieriger zu verstehen als ausführliche Strukturen (vgl. Härtig et al. 2019, S. 281-284).

Zur Unterscheidung von Alltags- und Bildungssprache hat Cummins (1979) zentrale Beiträge geleistet. Er differenziert in seinen Untersuchungen zwischen zwei sprachlichen Registern: alltagssprachliche dialogische Sprachkompetenz (BICS - Basic Interpersonal Communication Skills) und kognitiv-akademische Sprachkompetenz (CALP - Cognitive Academic Language Proficiency) (vgl. Cummins, Jim 1979, S. 198). BICS ist Alltagssprache und meint damit die Befähigung in alltäglichen Situationen, kontextgebunden und konzeptionell mündlich zu kommunizieren. Die Bezeichnung CALP hingegen ist das Gegenteil und wird synonym zur Bildungssprache verwendet (vgl. Siebert-Ott 2001, S. 32).

Alltags- und Bildungssprache sind nicht immer klar voneinander abzugrenzen. Sie werden außerdem in der Literatur unterschiedlich definiert und es existieren verschiedene Begriffe parallel nebeneinander. Zusammenfassend kann festgehalten werden, dass Alltagssprache in der Tendenz eher in alltäglichen Kontexten, konzeptionell mündlich und vorwiegend mit Kommunikationspartner*innen, die einem*r vertraut sind, verwendet wird, wohingegen in Abgrenzung dazu Bildungssprache in formellen Kontexten gebraucht wird, konzeptionell an 
Schriftsprache angelehnt ist und sich durch eine größere emotionale Distanz zwischen den Sprecher*innen auszeichnet. Dadurch, dass Alltagssprache im Vergleich zu Bildungssprache in der Literatur häufig als ungenau definiert wird, wird Bildungssprache allgemein zu Ungunsten der Alltagssprache als die entwickelte, ausgereifte Variante der Alltagssprache herausgestellt (vgl. Gantefort 2013, S. 72; Ortner 2009, S. 2227). Diese Auffassung wird in der vorliegenden Arbeit nicht geteilt. Denn schließlich entscheiden Funktion und Situation darüber, welches sprachliche Register angemessen ist. Wenn die Bedürfnisse einer Situation in Alltagssprache erfüllt werden, so passt diese genau und ist anderen Registern vorzuziehen (vgl. Höttecke et al. 2017, S. 3; Rincke 2010, S. 246).

\subsubsection{Bildungssprache vs. Fachsprache}

Bildungs- und Fachsprache teilen zentrale Charakteristika, weswegen eine Abgrenzung voneinander nicht trennscharf ist. Anhand der Kategorien field, mode und tenor zeigt sich, dass Bildungs- und Fachsprache eher ein Spektrum bilden und keine abgeschlossenen Begriffe sind.

\section{Bezugsbereich (field)}

Während Fachsprachen in der Linguistik in der Regel über die Zuordnung zu einem konkreten Fach definiert werden, fungiert Bildungssprache fächerübergreifend. Das, was Fachsprachen am eindeutigsten kennzeichnet, ist,,das Fach, der Gegenstand, das Thema, über das kommuniziert wird“" (Adamzik 1998, 184). Der Bezugsbereich beschränkt sich dabei auf die „wissenschaftlichen oder (berufs-) technischen Fachbereiche" (Riebling 2013, S. 117). Bildungssprache hingegen weist genau diese Fächergrenzen nicht auf, sondern bildet ein Scharnier zwischen Alltags- und Fachsprache, sodass ein Austausch zwischen den Registern möglich ist; sie dient im Wesentlichen fachexterner oder vermittelnder fachinterner Kommunikation.

\section{Medium/Konzeption (mode)}

Hinsichtlich der mode-Kategorie lassen sich keine klaren Grenzen zwischen Bildungssprache auf der einen und Fachsprache auf der anderen Seite ziehen. Eine Anordnung zwischen den Polen konzeptionell mündlich und konzeptionell schriftlich zeigt Tendenzen; die Grauabstufung weist darauf hin, dass „die Register keine hermetischen Systeme darstellen, sondern eine Reihe von Übergangsformen kennen“ (Riebling 2013, S. 121) (vgl. Abb. 2.2). 


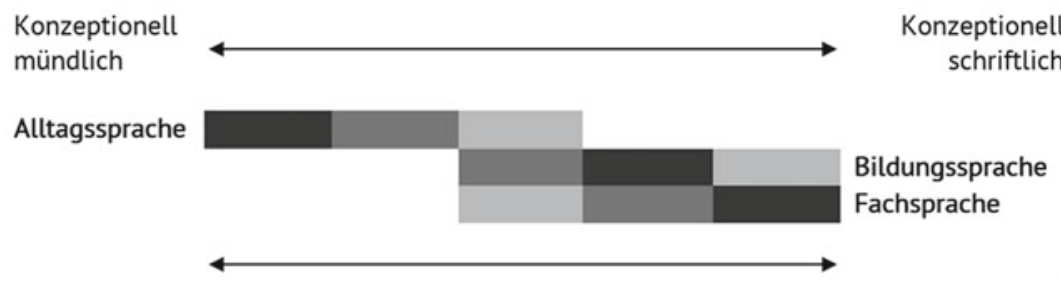

Abb. 2.2 Schematische Anordnung der Alltags-, Bildungs- und Fachsprache zwischen den Polen konzeptioneller Mündlichkeit und Schriftlichkeit. (Quelle: verändert nach Riebling 2013, S. 121)

\section{Interaktionssituation (tenor)}

Die Interaktionssituationen in Bildungs- und Fachsprache decken sich; beide finden in öffentlich-institutionellem Rahmen statt, wobei das Verhältnis der Gesprächspartner*innen eher durch emotionale Distanz und Fremdheit bestimmt ist. Insbesondere im Kontext der Schule zeichnen sich die Sprechenden durch Asymmetrien im Wissens- und Kompetenzstand aus. Die Lehrperson ist den Schüler*innen in der Regel bildungs- und fachsprachlich voraus.

Bildungs- und Fachsprache unterscheiden sich nur hinsichtlich ihres Bezugsbereichs eindeutig, während in den anderen Kategorien nur Unterscheidungstendenzen oder sogar Übereinstimmungen auszumachen sind. Auf sprachlicher Ebene zeigen sich daher am deutlichsten Unterschiede auf lexikalischer Ebene in Form von Fachbegriffen (vgl. Köhne et al. 2015, S. 69). Die Merkmale von Fachund Bildungssprache sind wie das Konstrukt Bildungs- und Fachsprache von Autor*in zu Autor*in unterschiedlich festgelegt. In einer umfangreichen Auflistung fassen Wildemann/Fornol (2016, S. 114-120) die verschiedenen Merkmale von Bildungssprache aus unterschiedlichen Quellen zusammen. Die Merkmale betreffen jeweils unterschiedliche Teilbereiche der allgemeinen Linguistik (vgl. Bußmann/Gerstner-Link 2002), insbesondere Syntax (Aufbau von Wortgruppen und Sätzen), Semantik (Bedeutung von Wörtern und Sätzen), Morphologie (Aufbau von Wörtern und Wortformen) sowie Lexik (Wortschatz). Ausgeklammert werden Phonologie (Lautsystem der Sprache) sowie Orthographie (Schriftsystem der Sprache). Auf den Bereich der Pragmatik (Bedeutung sprachlicher Äußerungen und ihre Anwendung) sowie diskurslinguistische Aspekte wird als Exkurs im folgenden Abschnitt eingegangen (vgl. Abschn. 2.1.3); denn dieser Teilbereich wird in der Debatte um Fachsprache noch selten genannt. Nachfolgend sind diejenigen Merkmale aufgezählt, die in diversen Quellen übereinstimmend als Merkmale für Bildungssprache genannt werden (vgl. Tab. 2.2). 
Tab. 2.2 Übersicht über Merkmale von Bildungssprache

\begin{tabular}{|c|c|}
\hline Passivgebrauch & $\begin{array}{l}\text { Ahrenholz 2013; Bailey 2006; Biber 1988; Eckhardt 2008; } \\
\text { Feilke 2012a; Gogolin et al. 2007; Heppt et al. 2012; Jeuk } \\
\text { 2010; Kniffka/Roelcke 2016; Luchtenberg 1988; Maas 2010; } \\
\text { Ohm et al. 2007; Quehl/Scheffler 2008; Reich 2008; Rincke } \\
\text { 2010; Roelcke 2020; Rösch 2005; Schmölzer-Eibinger 2013; } \\
\text { Steinmüller/Schwarnhorst } 1987\end{array}$ \\
\hline Relativsätze & $\begin{array}{l}\text { Ahrenholz 2013; Bailey 2006; Biber 1988; Quehl/Scheffler } \\
\text { 2008; Reich 2008; Schleppegrell 2001, 2006; } \\
\text { Steinmüller/Schwarnhorst } 1987\end{array}$ \\
\hline Komplexe Attribute & $\begin{array}{l}\text { Ahrenholz 2013; Feilke 2012a; Gogolin et al. 2007; Hempel } \\
\text { et al. 2019; Luchtenberg 1988; Maas 2010; Ohm et al. 2007; } \\
\text { Ortner 2009; Reich 2008; Rincke 2010; Roelcke 2020; } \\
\text { Schmölzer-Eibinger 2013 }\end{array}$ \\
\hline Partizipialkonstruktionen & $\begin{array}{l}\text { Ahrenholz 2013; Biber 1988; Jeuk 2010; Koch/Oesterreicher } \\
\text { 1985, 1994; Leckie-Tarry 1995; Ohm et al. 2007; Ortner } 2009\end{array}$ \\
\hline Funktionsverbgefüge & $\begin{array}{l}\text { Feilke 2012a; Jeuk 2010; Luchtenberg 1988; Reich 2008; } \\
\text { Roelcke 2020; Schmölzer-Eibinger } 2013\end{array}$ \\
\hline Nominalisierungen & $\begin{array}{l}\text { Bailey 2006; Biber 1988; Fang et al. 2006; Feilke 2012a; } \\
\text { Gibbons 2010; Gogolin et al. 2007; Heppt et al. 2012; Jeuk } \\
\text { 2010; Koch/Oesterreicher 1985, 1994; Leckie-Tarry 1995; } \\
\text { Luchtenberg 1988; Ortner 2009; Rincke 2010; Rösch 2005; } \\
\text { Schleppegrell 2001, 2006; Schmölzer-Eibinger 2013; } \\
\text { Steinmüller/Schwarnhorst } 1987\end{array}$ \\
\hline Hypotaxen & $\begin{array}{l}\text { Bailey 2006; Heppt et al. 2012; Koch/Oesterreicher 1985, } \\
\text { 1994; Leckie-Tarry 1995; Luchtenberg 1988; Ortner 2009; } \\
\text { Schmölzer-Eibinger } 2013\end{array}$ \\
\hline Fachbegriffe & $\begin{array}{l}\text { Bailey 2006; Eckhardt 2008; Feilke 2012a; Gogolin et al. } \\
\text { 2007; Heppt et al. 2012; Koch/Oesterreicher 1985, 1994; } \\
\text { Leckie-Tarry 1995; Luchtenberg 1988; Nodari/Steinmann } \\
\text { 2008; Ohm et al. 2007; Ortner 2009; Reich 2008; Rincke } \\
\text { 2010; Schleppegrell 2001, 2006; Schmölzer-Eibinger 2013; } \\
\text { Steinmüller/Schwarnhorst 1987; }\end{array}$ \\
\hline Präpositionen & $\begin{array}{l}\text { Bailey 2006; Eckhardt 2008; Feilke 2012a; } \\
\text { Koch/Oesterreicher 1985, } 1994\end{array}$ \\
\hline Konnektoren & $\begin{array}{l}\text { Ahrenholz 2013; Biber 1988; Eckhardt 2008; Gogolin et al. } \\
\text { 2007; Jeuk 2010; Koch/Oesterreicher 1985, 1994; } \\
\text { Leckie-Tarry 1995; Ohm et al. 2007; Quehl/Scheffler 2008; } \\
\text { Roelcke 2020; Schleppegrell 2001, } 2006\end{array}$ \\
\hline
\end{tabular}


Tab. 2.2 (Fortsetzung)

\begin{tabular}{l|l}
\hline Komposita & Eckhardt 2008; Feilke 2012a; Fuhrhop/Olthoff 2019; Gogolin \\
& et al. 2007; Leckie-Tarry 1995; Luchtenberg 1988; Ohm et al. \\
& 2007; Quehl/Scheffler 2008; Reich 2008; Rincke 2010; \\
& Roelcke 2020; Rösch 2005; Schmölzer-Eibinger 2013; \\
& Steinmüller/Schwarnhorst 1987 \\
\hline Pronomen, Adverbien & Eckhardt 2008; Feilke 2012a; Jeuk 2010; Quehl/Scheffler \\
& 2008
\end{tabular}

Quelle: verändert nach Fornol 2020, S. 67; Wildemann/Fornol 2016, S. 114-120

\subsubsection{Exkursorische Überlegungen zur Notwendigkeit von Fachsprache}

Es kann kritisch und grundsätzlich die Frage gestellt werden, inwiefern es anhand der aufgelisteten Merkmale überhaupt möglich ist, Aussagen über die fachsprachliche Kompetenz zu treffen. Ist gutes fachsprachliches Handeln nur dann möglich, wenn eine Reihe der oben genannten Merkmale erfüllt ist? Mehrere Autor*innen weisen in diesem Kontext auf die Notwendigkeit hin, die pragmatische und diskursive Ebene von Fachsprache und damit verbunden die Sprachsoziologie nicht aus dem Blick zu verlieren (vgl. Fornol 2017; Morek/Heller 2012; Oleschko 2017a). Denn auch Fachsprache sollte angemessen verwendet werden. In diesem Kontext wird die soziale Funktion von Fachsprache ,als Eintritts- und Visitenkarte“ (Morek/Heller 2012, S. 76) beleuchtet, die in den obigen Ausführungen zu Bernstein (1971) impliziert ist. Um diese soziale Dimension besser zu verstehen, hilft ein knapper Exkurs in die Arbeiten des Soziologen Bourdieu (1982). Er unterscheidet verschiedene Arten von Kapital. Neben dem klassischen ökonomischen Kapital (z. B. Geld, Aktien, Schmuck) benennt er soziales (z. B. Beziehungen), symbolisches (z. B. Prestige) und kulturelles Kapital als entscheidende Kriterien für Schichtzugehörigkeit. Kulturelles Kapital umfasst dabei die Fähigkeit zur guten, gewählten Rhetorik. Bourdieu zufolge ist der Sprachgebrauch status- und klassenspezifisch und reproduziert Bildungsungleichheiten. Dass große Schulleistungsstudien wie PISA, TIMSS und IGLU den sozioökonomischen Status beispielsweise relativ zuverlässig über die Anzahl der Bücher in einem Haushalt erheben, macht diesen Zusammenhang zwischen Sprache und Kapital und damit auch den Titel „Bildungssprache als Eintritts- und Visitenkarte“ offensichtlich. Man könnte Bildungs- und Fachsprache auch 
[...] als ,Geheimsprache" der Bildungs- und Lebenschancen zuteilenden Institution Schule bzw. als ihr eigentliches, aber geheimes Curriculum sehen, das bislang kaum transparent und eindeutig kodifiziert ist und an dem sich viele Lernende mächtig reiben oder gar scheitern. (Vollmer/Thürmann 2010, S. 109)

Vor diesem Hintergrund erscheint die Frage berechtigt, ob nicht eher eine Abkehr von Fachsprache zielführend ist auf dem Weg hin zu mehr Chancengleichheit. Ist es nachhaltiger, die ausschließenden, bildungssprachlichen Kreise aufzulösen, statt alle dazu befähigen zu wollen, sich in diesen Kreisen bewegen zu können? Letztlich wird es zur Überwindung der Diskrepanzen Anstrengungen aus beiden Richtungen brauchen. Schüler*innen sollten, so sie wollen, Zugang zu Fachsprache haben, sie verstehen und als Kulturwerkzeug verwenden können. Denn jedenfalls bis zu einem bestimmten Grad ist Fachsprache notwendig. Sie ist, wie oben aufgeführt, präzise, korrekt und führt dazu, dass sich Menschen derselben Community fachlich angemessen verständigen können. Auch die verschiedenen, in der Einleitung aufgeführten Studien, die den Zusammenhang zwischen Sprache und Fachkompetenz nahelegen sowie die noch folgenden Ausführungen zur Studienlage deuten darauf hin, Sprache wegen ihrer erkenntnisschaffenden Funktion unbedingt im Fachunterricht zu adressieren. Außerdem ist die Schule wegen des prinzipiell egalitären Charakters der Schulpflicht grundsätzlich ein geeigneter Ort, um Unterschiede aufzuweichen (vgl. Mafaalani 2020, S. 15). Darüber hinaus ist Fachsprache ein nicht zu unterschätzendes gruppenstiftendes Element, das zu einem Gefühl von Zusammengehörigkeit führt. Andererseits ist es genau dieses gruppen- und identitätsstiftende Charakteristikum von Fachsprache, das ausschließt. Denn Mitglied einer Gruppe zu sein, bedeutet auch, nicht zu einer anderen zu gehören. Die Kriterien, die zu Gruppenzugehörigkeit oder -ausschluss führen, müssen immer wieder hinterfragt und problematisiert werden. Wie sinnvoll oder -frei sind dabei bestimmte Kriterien? So sei dahingestellt, inwiefern es komplexe Satzstrukturen, eine hohe Dichte an Fachbegriffen, ungebräuchliche Verben und Adjektive oder passiven Satzbau braucht, um die Zugehörigkeit der sprechenden Person zu einer bestimmten Gruppe zu verdeutlichen. Letztlich geht es um adressant*innengerechtes sprachliches Handeln. Die Frage, wie das auszusehen hat, muss individuell und kontextabhängig immer wieder neu beantwortet werden. Genau in dieser Frage (Wie schreibe/spreche ich für wen?) liegt eine weitere Notwendigkeit für sprachsensiblen Fachunterricht. Denn um sich für oder gegen einen bestimmten Sprachstil entscheiden zu können, müssen die verschiedene Stile zunächst beherrscht werden. 


\subsubsection{Schul- und Unterrichtssprache im Gefüge von Alltags-, Bildungs- und Fachsprache}

Im Rahmen der vorliegenden Arbeit werden ausschließlich die funktionalsituativen Varietäten, die im Kontext Schule relevant sind, betrachtet, sprich: Alltags-, Bildungs- und Fachsprache. ${ }^{4}$ Bei manchen Autor*innen ist zusammenfassend von Schul- und/oder Unterrichtssprache die Rede, die heterogen und teils widersprüchlich definiert sind (vgl. u. a. Feilke 2012b). Der Begriff Schulsprache (Schleppegrell 2004) wird nachfolgend aufgrund der Unklarheit über den eigentlichen Bezugsbereich nicht verwendet. Die Unterrichtssprache, auf die Bezug genommen wird, ist Deutsch. Nur wenn die Unterrichtssprache ausreichend beherrscht wird, kann überhaupt fachlicher Wissenserwerb stattfinden (vgl. Fornol 2020, S. 11). Für die einzelnen sprachlichen Varietäten werden die eingeführten Begriffe von Alltags-, Bildungs- und Fachsprache im Kontext der Schule verwendet. Es wurden ausführlich Unterschiede und Gemeinsamkeiten von Alltags-, Bildungs- und Fachsprache dargelegt; daraus resultieren als Zusammenfassung dieses Kapitels die folgenden Definitionen.

Alltagssprache wird vorwiegend in alltäglichen Kontexten verwendet und zeichnet sich durch konzeptionelle Mündlichkeit aus, wie es für Gespräche in gesprochener Sprache mit (gut) bekannten Kommunikationspartner*innen üblich ist.

Bildungssprache dient der Kommunikation in öffentlich-formellen Kontexten. Das, worüber gesprochen wird, findet nicht im unmittelbaren und gemeinsamen Erlebniskontext der einander meist weniger bekannten Sprecher*innen statt, sodass komplexere sprachliche Mittel und Strukturen der Schriftsprache verwendet werden müssen, um die Distanz zwischen Gesagtem und eigentlich Gemeintem zu überbrücken. Die zentrale Funktion von Bildungssprache ist die Vermittlung zwischen alltäglichen und fachlichen Inhalten.

\footnotetext{
${ }^{4}$ Selbstredend wird im Rahmen der Schule auch von regionalen und sozialen Varietäten (diatopische und diastratische Dimensionen) Gebrauch gemacht. Fokussiert werden in der vorliegenden Arbeit nur funktional-situative Varietäten (diaphasische Dimension), also sprachliche Register.
} 
In der Fachsprache bezieht sich der Kommunikationsgegenstand nicht auf den gemeinsamen und unmittelbaren Erlebniskontext, sodass sprachliche Mittel und Strukturen der Schriftsprache zum Verständnis verwendet werden müssen. Zentraler Unterschied zur Bildungssprache ist, dass der Kommunikationsinhalt durch das entsprechende Fach begrenzt wird; daraus ergeben sich insbesondere Unterschiede auf lexikalischer Ebene.

\subsection{Die Rolle(n) von Sprache(n) im Geographieunterricht}

Sprache hat, wie die dargelegten Ausführungen zeigen, in ihren verschiedenen Varietäten unterschiedliche Ausprägungen und Funktionen, die sich auch im Geographieunterricht zeigen. Doch welche Funktionen hat Sprache im Geographieunterricht im Einzelnen? Sprache als ,die Fähigkeit zu sprechen“ (Dudenredaktion 2011, S. 876) fungiert in zweierlei Form. Zum einen dient sie der Kommunikation zwischen Individuen, ist also Medium (vgl. Becker-Mrotzek/Quasthoff 1998, S. 5). Zum anderen ist sie kognitive Leistung, die geübt werden muss (vgl. Volmert 2005, S. 13) und somit sowohl ein Lernziel als auch ein mögliches Lernhindernis des Geographieunterrichts. Mit Blick auf die verschiedenen Rollen von Sprache sind diskontinuierliche Darstellungsformen insofern besonders beachtenswert, als diese die verschiedenen Rollen von Sprache im Geographieunterricht miteinander verbinden. Diese verschiedenen Rollen stelle ich nachfolgend in der Theorie sowie im Licht der geographiedidaktischen Forschung dar.

\subsubsection{Sprache als Lernziel}

Seit dem PISA-Schock im Jahr 2000 (Baumert et al. 2006b), in dem die im internationalen Vergleich schlechten Leistungen deutscher Schüler*innen offensichtlich wurden, ist Sprache auch in einigen Bildungsstandards und Curricula natur- und gesellschaftswissenschaftlicher Fächer verankert. Der Kompetenzbereich Kommunikation ist in den Nationalen Bildungsstandards im Fach Geographie festgeschrieben. Die Schüler*innen sollen dazu befähigt werden, geographische Sachverhalte zu verstehen und sich sachgerecht auszudrücken (K1) sowie mit anderen in Interaktion zu treten (K2): 
K1 Fähigkeit, geographisch/geowissenschaftlich relevante Mitteilungen zu verstehen und sachgerecht auszudrücken.

Schülerinnen und Schüler können

- $S^{5} 1$ geographisch relevante schriftliche und mündliche Aussagen in Alltags- und Fachsprache verstehen,

- S2 geographisch relevante Sachverhalte/Darstellungen (in Text, Bild, Grafik etc.) sachlogisch geordnet und unter Verwendung von Fachsprache ausdrücken,

- S3 bei geographisch relevanten Aussagen zwischen Tatsachenfeststellungen und Bewertungen unterscheiden,

- S4 geographisch relevante Mitteilungen fach-, situations- und adressatengerecht organisieren und präsentieren.

K2 Fähigkeit, sich über geographische/geowissenschaftliche Sachverhalte auszutauschen, auseinanderzusetzen und zu einer begründeten Meinung zu kommen.

Schülerinnen und Schüler können

- S5 im Rahmen geographischer Fragestellungen die logische, fachliche und argumentative Qualität eigener und fremder Mitteilungen kennzeichnen und angemessen reagieren,

- S6 an ausgewählten Beispielen fachliche Aussagen und Bewertungen abwägen und in einer Diskussion zu einer eigenen begründeten Meinung und/oder zu einem Kompromiss kommen (z. B. Rollenspiele, Szenarien).

(DGfG 2017, S. 22-23)

Budke/Kuckuck (2017a) betrachten die von den Nationalen Bildungsstandards im Fach Geographie geforderten sprachlichen Kompetenzen vor dem Gemeinsamen Europäischen Referenzrahmen für Sprachen ${ }^{6}$. Sie unterscheiden für den Bereich Sprachkompetenz die für den Geographieunterricht relevanten Dimensionen Sprachrezeption, Sprachinteraktion und Sprachproduktion sowohl im Mündlichen als auch im Schriftlichen (vgl. Europarat 2001, S. 62-91). Bezogen auf den Geographieunterricht meint Sprachrezeption, dass Schüler*innen fachspezifische Darstellungen wie z. B. Klimadiagramme, topographische Karten, Texte, Filme, aber auch Unterrichtsgespräche verstehen können. Die Kompetenzdimension Sprachproduktion meint, dass Schüler*innen geographische Phänomene,

\footnotetext{
${ }^{5} \mathrm{~S}=$ Standard

${ }^{6}$ Der gemeinsame Europäische Referenzrahmen für Sprachen (GER) erfüllt eine Vielzahl von Funktionen und dient zwischenzeitlich vordergründig zur Beurteilung von Lernfortschritten in einer Fremdsprache. Ziel ist die bessere Vergleichbarkeit europäischer Sprachzertifikate durch Maßstäbe für den Erwerb von Sprachkenntnissen. Der GER wird als Referenzrahmen herangezogen, da er die verschiedenen Anforderungsbereiche an Sprache vor dem Hintergrund der multilingualen Schulrealität sinnvoll aufschlüsselt.
} 
Probleme und Zusammenhänge in angemessener Form mündlich und schriftlich versprachlichen können. Die Dimension der Sprachinteraktion bezieht sich auf den mündlichen oder schriftlichen Austausch über geographische Fachinhalte zwischen mindestens zwei Personen (vgl. Budke/Kuckuck 2017a, 16).

Durch die Festschreibung des Kompetenzbereichs Sprache in den Nationalen Bildungsstandards des Faches Geographie findet sich dieser auch in den circa 50 verschiedenen deutschen Curricula für den Geographieunterricht (verschiedene Klassenstufen und Schulformen in 16 verschiedenen Bundesländern) wieder; wenn auch in unterschiedlichem Ausmaß und unterschiedlicher Komplexität. Budke/Weiss (2014) haben die Lehrpläne hinsichtlich der Sprachkompetenz analysiert und herausgestellt, dass Sprache als Lernziel häufig implizit vorhanden ist, da es als Medium für sehr unterschiedliche Anforderungen benutzt wird. Explizit wird Sprache als Lernziel insbesondere in der Forderung nach dem Erwerb von Fachbegriffen. Die Schüler*innen sollen ihren Fachwortschatz ausbauen und die gelernten Fachbegriffe in einen inhaltlichen Kontext einbinden sowie zur Beantwortung geographischer Fragestellungen nutzen. Ferner sollen sie in der Lage sein, Fachsprache in Alltagssprache zu übersetzen (vgl. Budke/Weiss 2014, S. 114-116). Hofmann et al. (2012) stellen in einem Beitrag zum konstruktivistischen Raumverständnis die besondere Bedeutung von Sprache zur Reflexion über Raum dar. Grundtenor ist, dass Raum nicht normativ gesetzt ist, sondern durch Bedeutungszuschreibungen - (auch) über Sprache - gemacht wird (vgl. Hofmann et al. 2012, S. 50). Dies ist ein zentraler Gedanken bei der Diskussion um Sprache als Lernziel in einem konstruktivistischen Verständnis von Geographie(lernen) und Raum, der durch Kommunikation entsteht ${ }^{7}$ (vgl. Schwarze 2019, S. 115).

\subsubsection{Sprache als Lernmedium}

Der Erwerb geographischer Kompetenzen ist nicht sprachfrei möglich. Dies ist insofern wenig überraschend, als Sprache das zentrale Medium des Geographieunterrichts und des Fachunterrichts im Allgemeinen ist (vgl. Felder 2006, S. 42). Fachliche Informationen werden sprachlich vermittelt, Unterrichtsmedien werden

\footnotetext{
${ }^{7}$ Die allgemeine Skepsis gegenüber Sprache als eindeutigem, normiertem Medium kam mit der konstruktivistischen Wende auf und wird unter dem Begriff linguistic turn gefasst. Sprache wird demnach nicht länger nur als Medium gesehen, das die Wirklichkeit objektiv erfasst; viel eher wird ,alle menschliche Erkenntnis durch Sprache strukturiert“ (Nünning 2004, S. 147) und somit das Denken durch Sprache entsprechend beeinflusst.
} 
unter Nutzung der Sprache ausgewertet, Diskussionen über fachliche Zusammenhänge geführt, Arbeitsergebnisse formuliert und Lernfortschritte ebenfalls sprachlich kommuniziert (vgl. Michalak et al. 2015, S. 13). Sprache ist damit die Grundlage des Erwerbs fachlicher Inhalte und gleichzeitig die zu erwerbende Grundlage, um fachlich angemessen zu kommunizieren.

Für das Fach Geographie zeigt sich, dass die Informationsaufnahme über verschiedene textliche und nicht-textliche Darstellungsformen erfolgt. Ein Fokus der Informationsdarstellung liegt auf Sachtexten, die gelesen, verstanden und ausgewertet werden sollen. Die Bedeutung von Sprache schreibt sich im Unterrichtsgespräch fort. Schüler*innen sollen Argumente verstehen und bewerten können sowie unter Beachtung der eigenen Subjektivität selbst Argumente in einer Auseinandersetzung entwickeln und vertreten; das alles ist ohne Sprache nicht möglich. Dies gilt in gleichem Maße für die Präsentation von Arbeitsergebnissen, die sprachlich präzise und korrekt umgesetzt werden sollen. Ein weiterer zentraler Bereich des Geographieunterrichts ist die kritische Reflexion über genutzte Informationen und Medien, aber auch über Unterrichtsergebnisse und den eigenen Lernprozess, die eigene Meinung oder eigene Argumentationsketten. Auch hierzu muss sich wesentlich und auf ausreichend hohem Niveau der Sprache bedient werden (vgl. Budke/Weiss 2014, S. 114-116). In diesem Zusammenhang spielen in den deutschen Lehrplänen Operatoren, die zu kognitiven und sprachlichen Handlungen auffordern, eine zentrale Rolle (vgl. Thürmann 2012, S. 5). Fachübergreifend wichtige Sprachhandlungen im Unterricht sind unter anderem beschreiben, erklären, vergleichen, analysieren und interpretieren (vgl. Michalak et al. 2015, S. 52).

Mit der Formulierung von Operatorenlisten für zentrale Prüfungen und mit der Verwendung von Operatoren in der Unterrichtspraxis ist das Ziel verbunden, Klarheit darüber zu schaffen, wie eine Aufgabe zu bearbeiten ist. Während Fragen höchstens implizit zum Ausdruck bringen, in welcher Form sie beantwortet werden sollen, und während bestimmte, geschlossene Fragetypen leicht auf Ein-Wort-Antworten oder eine bloße Wissensabfrage hinauslaufen, besteht mit der Formulierung von Aufgaben mit Operatoren die Möglichkeit, die mit ihnen verbundenen inhaltlichen und sprachlichen Anforderungen klarer und transparenter darzustellen. Allerdings ist diese Transparenz keineswegs automatisch gegeben, und es ist notwendig, bei der Formulierung von Aufgabenstellungen weitere, oft auch fachspezifische Präzisierungen vorzunehmen sowie in einem differenzierenden und sprachfördernden Unterricht Unterstützungsangebote bereitzustellen. (Peuschel/Burkard 2019a, S. 56)

Die Operatoren haben fachspezifische Ausprägungen. So unterscheidet sich beispielsweise der Operator begründen von Fach zu Fach teilweise wesentlich (vgl. Tab. 2.3). 
Tab. 2.3 Der Operator begründen in den Einheitlichen Prüfungsanforderungen (EPA) ausgewählter Fächer

\begin{tabular}{l|l}
\hline Geographie & $\begin{array}{l}\text { Aussagen (zum Beispiel eine Behauptung, eine Position) durch } \\
\text { Argumente stützen, die durch Beispiele oder andere Belege } \\
\text { untermauert werden }\end{array}$ \\
\hline Ethik & $\begin{array}{l}\text { einen Sachverhalt oder eine Aussage durch nachvollziehbare } \\
\text { Argumente stützen }\end{array}$ \\
\hline Ev. Religion & Aussagen durch Argumente stützen \\
\hline Geschichte & $\begin{array}{l}\text { Aussagen (z. B. Urteil, These, Wertung) durch Argumente } \\
\text { stützen, die auf historischen Beispielen und anderen Belegen } \\
\text { gründen }\end{array}$ \\
\hline Philosophie & $\begin{array}{l}\text { Hinsichtlich der Ursachen und Folgerungen schlüssige } \\
\text { Zusammenhänge ausführlich und differenziert darlegen }\end{array}$ \\
\hline Sozialkunde/ Politik & $\begin{array}{l}\text { Zu einem Sachverhalt komplexe Grundgedanken unter dem } \\
\text { Aspekt der Kausalität argumentativ und schlüssig entwickeln }\end{array}$ \\
\hline
\end{tabular}

Quelle: verändert nach Peuschel/Burkard 2019a, S. 57

Die verschiedenen Operatoren werden in den Curricula drei Anforderungsbereichen (AFB) zugeordneten. Für das Fach Geographie umfasst der Anforderungsbereich I Reproduktionsleistung, Anforderungsbereich II erfordert Reorganisations- und Transferleistung und der dritte Bereich bezieht sich auf Leistungen der Reflexion und Problemlösung (KMK 2005). Ebenso wie die Definition der Operatoren selbst, ist auch deren Zuordnung zu Anforderungsbereichen nicht in jedem Fach gleich.

In den EPA für die Fächer Geographie, Geschichte, Philosophie, Sozialkunde/Politik und Wirtschaft werden zum Beispiel die Operatoren beschreiben und wiedergeben jeweils dem AFB I zugeordnet, analysieren und erläutern zählen zum AFB II, während beurteilen, entwickeln und Stellung nehmen dem AFB III zugeordnet werden. Daneben gibt es aber auch Operatoren, die zwei oder sogar allen drei Anforderungsbereichen zugeordnet sind, und zwischen den Fächern bestehen teils Unterschiede in den Zuordnungen. So wird zum Beispiel darstellen im Fach Sozialkunde/Politik nur dem AFB I zugeordnet, im Fach Philosophie hingegen den AFB I und II. Interpretieren im Fach Geschichte und eine philosophische Problemreflexion durchführen im Fach Philosophie verlangen jeweils Leistungen in allen drei Anforderungsbereichen. (Peuschel/Burkard 2019a, S. 56)

Die unterschiedlichen Definitionen der Operatoren sowie deren heterogene Zuordnung zu den Anforderungsbereichen in den Fächern legt erneut eine explizite fachspezifische Adressierung von Sprache im Geographieunterricht nahe. 


\subsubsection{Sprache als mögliches Lernhindernis}

Sprache ist also ausgemachtes Lernziel in Curricula und Bildungsstandards und es ist auch das zentrale Medium, um genau diese sprachlichen sowie fachlichen Ziele zu erreichen. Doch die Anforderungen an das Lernen von Fachsprache (vgl. Abschn. 2.1) können ohne explizite Adressierung zum Lernhindernis werden.

Schüler*innen behandeln aktuelle gesellschaftliche Probleme häufig auch außerhalb der Schule in ihrem privaten Alltag und umgekehrt. Sowohl in der Alltags- als auch in der Unterrichtsrealität kann daher eine Vermischung der unterschiedlichen Register stattfinden. So, wie die Schüler*innen mit alltagssprachlich geprägtem Vorwissen in den Unterricht kommen, können sie umgekehrt z. B. auch fachliche Texte im Alltag lesen. Ein aktuelles Beispiel ist der Klimawandel, der im Geographieunterricht behandelt wird, über den sich viele Schüler*innen im privaten Kontext in Bewegungen wie Fridays for Future austauschen und der zentraler Gegenstand der Berichterstattung in sozialen und weiteren Medien ist. Die Schüler*innen verfügen also möglicherweise über Informationen, Meinungen und Formulierungen, die sie im Alltagskontext und in Alltagssprache erworben haben. Im Bildungskontext, zum Beispiel beim Lesen eines Fachtextes zum Klimawandel im Geographieunterricht, reichen die alltagssprachlichen Kompetenzen der Schüler*innen aber möglicherweise nicht mehr aus, um Inhalte angemessen $\mathrm{zu}$ verstehen und eigene Gedanken fachsprachlich angemessen $\mathrm{zu}$ versprachlichen. So gibt es teilweise Fachbegriffe, welche im Fachkontext eine andere Bedeutung haben, als aus dem Alltag bekannt, oder metaphorisch besetzt sind (z. B. Treibhauseffekt). Außerdem müssen die Schüler*innen die Fähigkeit erwerben, alle typischen im Geographieunterricht eingesetzten Medien, wie Texte, Grafiken, Karten, Statistiken etc., zu entschlüsseln.

\subsubsection{Diskontinuierliche Darstellungsformen und deren Vernetzung als Lernziel, -medium, -hindernis und -hilfe}

Eine besondere Rolle in der Diskussion um Sprache und Geographieunterricht nehmen diskontinuierliche Darstellungsformen ein, die sowohl als Lernziel und Lernhindernis, aber auch als Lernmedium und -hilfe fungieren können. Die hohe Anzahl an verschiedenen diskontinuierlichen Darstellungsformen, die Text mit Bild- und Diagrammelementen verbinden, stellen eine Besonderheit des Geographieunterrichts dar (vgl. Kuckuck 2019, S. 179). Zu ihnen gehören Karten, Klimadiagramme, Alterspyramiden, Höhenprofile, Experimente, Modelle, Schemata, Diagramme und Tabellen. All jene Darstellungsformen treten aber auch 
im Alltag, zum Beispiel in Zeitungen, Nachrichten oder sozialen Medien auf (vgl. Michalak et al. 2017, S. 78). In der Literatur werden der Wechsel und die Vernetzung verschiedener Darstellungsformen als besonders gewinnbringend für das fachliche Verständnis herausgestellt. Doch damit positive Effekte durch den Wechsel von Darstellungen entstehen können, müssen Lernende zunächst dazu befähigt werden, Darstellungsformen überhaupt angemessen zu verstehen und in weiterer Konsequenz zu versprachlichen. Bevor diskontinuierliche Darstellungsformen Lernhilfe sein können, ist ihr Begreifen zunächst Lernziel, damit sie kein Lernhindernis sind (vgl. Ainsworth et al. 2002, S. 25). Diskontinuierliche Darstellungsformen sind nicht selbsterklärend; die Lernenden müssen sie selbst aktiv deuten. Auch „Informationen aus Bildern und Bilderfolgen zu entnehmen, richtet spezifische Verstehensanforderungen an Rezipienten“ (Damman-Thedens/Michalak 2012, S. 139). Dies legt nahe, eine visual literacy in der Schule fächerübergreifend aktiv zu erwerben (vgl. Michalak 2012a, S. 109). Mit Diagrammen zum Beispiel werden Schüler*innen verstärkt erst an weiterführenden Schulen konfrontiert; sie wissen aus der Grundschulzeit noch nicht viel damit anzufangen (vgl. Stecken 2013, S. 299). Um den vielfältigen sprachlichen und fachlichen Anforderungen von diskontinuierlichen Darstellungsformen gerecht zu werden, schlagen Michalak/Müller (2015, 2016, 2017) sogenannte Sprach-Fach-Netze vor. Diese stellen eine sequenzierte Unterstützung dar. Dabei handelt es sich um ein vierphasiges Modell zur Analyse diskontinuierlicher Darstellungsformen. Das Modell beinhaltet standardisierte Fragen, die anhand verschiedener Erwartungshorizonte aus den Bildungsstandards entwickelt wurden. Es soll außerdem sprachliche Unterstützung gewährleisten. Die erste Phase dient der Orientierung, in der die Dokumentation zentraler Rahmendaten, wie beispielsweise Titel, Quelle, Ort und Zeit der Daten aus dem Diagramm aufgeführt werden. Die zweite Phase soll die genaue Betrachtung der enthaltenen Information eines Diagramms ermöglichen. In Phase drei erfolgt die Erklärung, in der mögliche Gründe, Ursachen und Folgen erläutert werden. Innerhalb dieser Phase müssen Kontextinformationen genutzt werden, die über die im Diagramm dargestellten Informationen hinausgehen. Die vierte Phase der Beurteilung/Bewertung beinhaltet zwei wesentliche Aspekte. Zum einen eine Bewertung bzw. Beurteilung des Diagramms und zum anderen eine Bewertung der dargestellten Inhalte (vgl. Michalak/Müller 2015, S. 153-154). Erste empirische Befunde zeigen eine positive Wirkung bei der Versprachlichung und dem Verständnis von diskontinuierlichen Darstellungsformen, obwohl Anschlussuntersuchungen noch ausstehen, da bisher eine zu geringe empirische Basis vorliegt (vgl. Michalak/Müller 2016, S. 47). 
Werden die Darstellungsformen verstanden, so können sie den fachlichen und sprachlichen Verstehensprozess im Geographieunterricht durch Darstellungsvernetzung unterstützen. Während für das Fach Mathematik diverse Studien den positiven Einfluss von Darstellungswechseln auf das Mathematiklernen herausstellen, gibt es für das Fach Geographie noch keine entsprechenden Untersuchungen. Da der Geographieunterricht allerdings ebenso wie der Mathematikunterricht mit einer Reihe von diskontinuierlichen Darstellungen arbeitet, ist anzunehmen, was Wittmann (1981, S. 91) für den Mathematikunterricht argumentiert: „Wissen, das in verschiedenen Darstellungen erworben wurde und verfügbar ist, kann leichter behalten werden und die Fähigkeit, Wissen nach Bedarf in die eine oder andere Form zu transponieren, erhöht die Flexibilität und den Erfolg beim Problemlösen“. Werden die Darstellungsformen in ihrer Struktur verstanden, so können sie eine große Lernhilfe sein, insbesondere dann, wenn die Kombination mehrerer Darstellungsformen durchgeführt wird. Dann kann eine Art Übersetzungstätigkeit zwischen realer Sachsituation, Experiment (vgl. Gottwald 2016; Scheuer et al. 2010, S. 92), Bild und der symbolischen Darstellung operationalisiert werden. Die Vernetzung der Darstellungen sollte also unbedingtes Lehrund Lernziel des Geographieunterrichts sein. Die Darstellungsvernetzung fungiert dann als Problemlöse- und Argumentationsmittel, als Lernmedium, ebenso wie als Begriffs- und Vorstellungsstütze (vgl. Wessel 2015, S. 72). Darüber hinaus ist die Verwendung unterschiedlicher Darstellungsformen ein Schlüssel zur fachlichen und sprachlichen Zusammenführung.

Der Wechsel der Darstellungsformen erweist sich als der didaktische Schlüssel zum fachlichen Verstehen und ist ein Anlass zur fachlichen Kommunikation. Es ist didaktisch klug, ja, sogar zwingend, diesen Wechsel der Darstellungsformen in das Zentrum der Didaktik des bilingualen Sachfachunterrichts zu stellen. Dadurch ist sichergestellt, dass an den Gegenständen und Belangen des Faches in originärer Weise gearbeitet wird. (Leisen 2005, S. 9)

Die wesentlichen Vorteile der Darstellungsvernetzung liegen in einer sprachlichen Entlastung durch Darstellungsvielfalt sowie der Entwicklung von Sprache und Vorstellungen durch den bewussten Einsatz grafischer und symbolischer Darstellung sowie die gezielten Wechsel der Register innerhalb der verbalen Darstellung (Wessel 2015, S. 77). Es wird also eine Vernetzung der Darstellungsformen angestrebt. Das Modell von Prediger/Wessel (2011), das zwischenzeitlich weiterentwickelt wurde, zeigt auf den verschiedenen Darstellungsebenen (symbolisch, verbal, bildlich, gegenständlich) auch die Untergliederung in Erstsprache (L1) und Zweitsprache (L2) und differenziert die verbale Ebene in die Register fachsprachlich, bildungssprachlich und alltagssprachlich. So wird einerseits auf 
die vielfältigen Zugangsmöglichkeiten und andererseits auf die möglicherweise sehr heterogenen Eingangsvoraussetzungen von Schüler*innen verwiesen (vgl. Abb. 2.3).

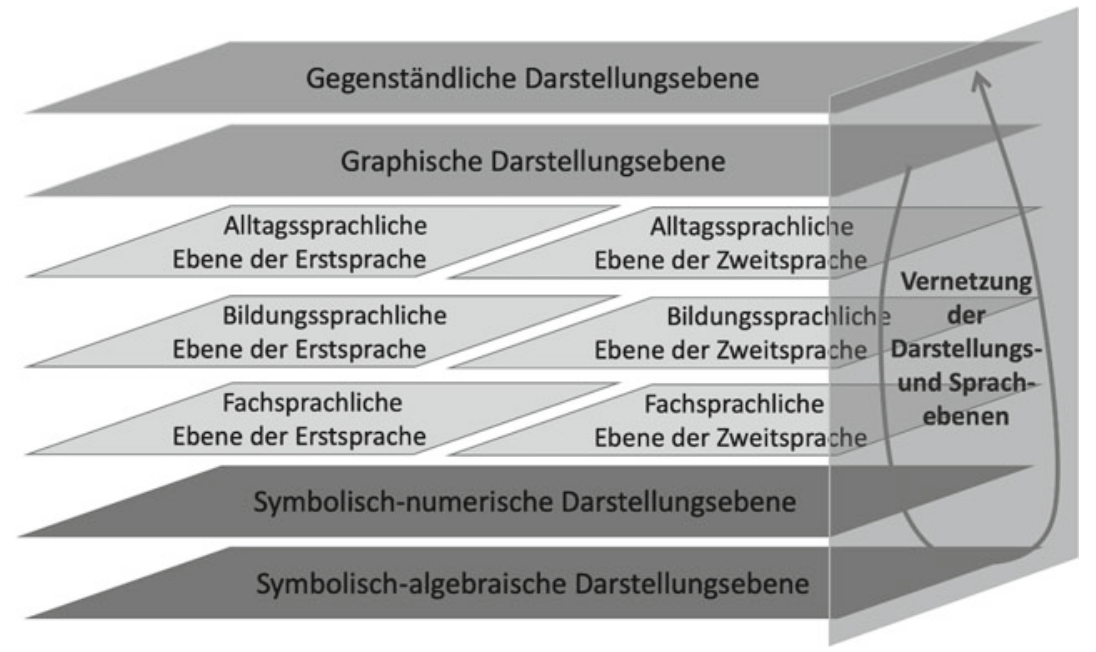

Abb. 2.3 Modell zur Vernetzung der Darstellungs- und Sprachebenen im Fachunterricht. (Quelle: Prediger 2020, S. 43)

Im Gegensatz zu Darstellungen anderer Autor*innen wird nicht hierarchisch streng zwischen den Ebenen unterschieden. Lediglich die verbale Darstellung in alltags-, bildungs- und fachsprachlich wird eindeutig in der Schwierigkeit hierarchisiert. „Die anderen werden als gleichwertig betrachtet und sind in Hinblick auf ihren Abstraktionsgrad gestuft [...]. Denn je nach Kontext und Thema können die Abstraktionsgrade variieren“ (Wessel 2015, S. 78-79). Die Darstellungsvernetzung stellt im Rahmen der vorliegenden Arbeit einen Kernpunkt für die Entwicklungsarbeit dar, da sie sowohl geographie- als auch sprachdidaktisch wertvoll erscheint. Für das Fach Biologie zum Beispiel hat sich die Verknüpfung von Text und Bild als entscheidend für den Aufbau fachspezifischer Kompetenzen erwiesen (vgl. Drumm 2017, S. 51). Die folgenden Punkte legen den Profit eines Darstellungswechsels im sprachsensiblen Fachunterricht aus interdisziplinärer Perspektive dar (vgl. Tab. 2.4).

Die nachfolgende Tabelle zeigt die verschiedenen Abstraktionsgrade von Sprache mit Beispielen für den Geographieunterricht aus der in der vorliegenden 
Tab. 2.4 Mehrwert von Darstellungswechseln

1. Fachlich: Es handelt sich um eine den Sachverhalten angemessene Darstellung.

2. Didaktisch: Ein Sachverhalt wird leichter und besser verstanden, wenn er über verschiedene Formen der Darstellung angegangen wird.

3. Methodisch: Ein Wechsel der Darstellungsformen ist motivierender.

4. Lernpsychologisch: Es werden mehrere Wahrnehmungskanäle benutzt.

5. Pädagogisch: Die Nutzung unterschiedlicher Darstellungsformen erlaubt eine ebenso angemessene wie leistbare Binnendifferenzierung und lässt die arbeitsteilige Bearbeitung in Gruppen zu.

6. Sprachdidaktisch: Fachliche Inhalte sind ohne sprachliche Darstellung verständlich; anhand nicht-sprachlicher Darstellungen kann fachlich angemessene Versprachlichung explizit geübt werden.

Quelle: verändert nach Leisen 2013, S. 37

Studie untersuchten, sprachsensiblen Unterrichtsreihe (vgl. Tab. 2.5). Dabei sind, wie auch für die Mathematikdidaktik, die unterschiedlichen Abstraktionsgrade abgebildet, wobei die abstrakten Darstellungen oft - aber nicht immer und nicht zwangsläufig - weniger intuitiv zugänglich sind als konkrete Darstellungen.

Tab. 2.5 Wechsel der Darstellungsform im Fach Geographie

\begin{tabular}{|c|c|c|c|c|c|c|c|}
\hline & Ebene & Sprache & \multicolumn{5}{|c|}{ Bsp.: Plattentektonik (Dichte) } \\
\hline \multirow[t]{3}{*}{$\begin{array}{l}\text { hoher } \\
\text { Abstraktionsgrad }\end{array}$} & mathematisch & $\begin{array}{l}\text { mathematische } \\
\text { Sprache: } \\
\text { Gesetze, } \\
\text { Formeln, } \\
\text { Maßeinheiten }\end{array}$ & \multicolumn{5}{|c|}{$\begin{array}{l}\text { Maßeinheiten } \\
\text { kontinentale Kruste }=2,7 \mathrm{~g} / \mathrm{cm}^{3} \\
\text { ozeanische Kruste }=3,0 \mathrm{~g} / \mathrm{cm}^{3}\end{array}$} \\
\hline & symbolisch & $\begin{array}{l}\text { Symbolsprache: } \\
\text { Diagramme, } \\
\text { Graphen } \\
\text { Tabellen }\end{array}$ & 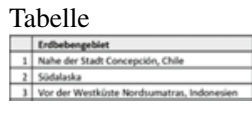 & & 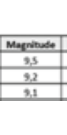 & 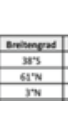 & 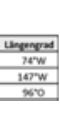 \\
\hline & sprachlich & $\begin{array}{l}\text { Fachsprache } \\
\text { oder } \\
\text { Alltagssprache } \\
\text { in Form von } \\
\text { Text, } \\
\text { Mind-Map, } \\
\text { Gliederung, } \\
\text { Definitionen }\end{array}$ & \multicolumn{5}{|c|}{ 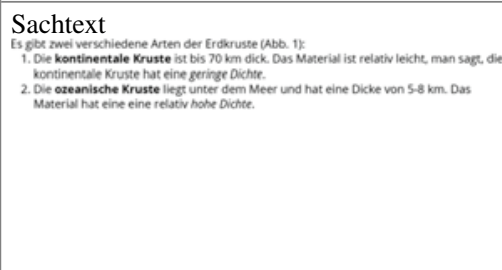 } \\
\hline
\end{tabular}

(Fortsetzung) 
Tab. 2.5 (Fortsetzung)

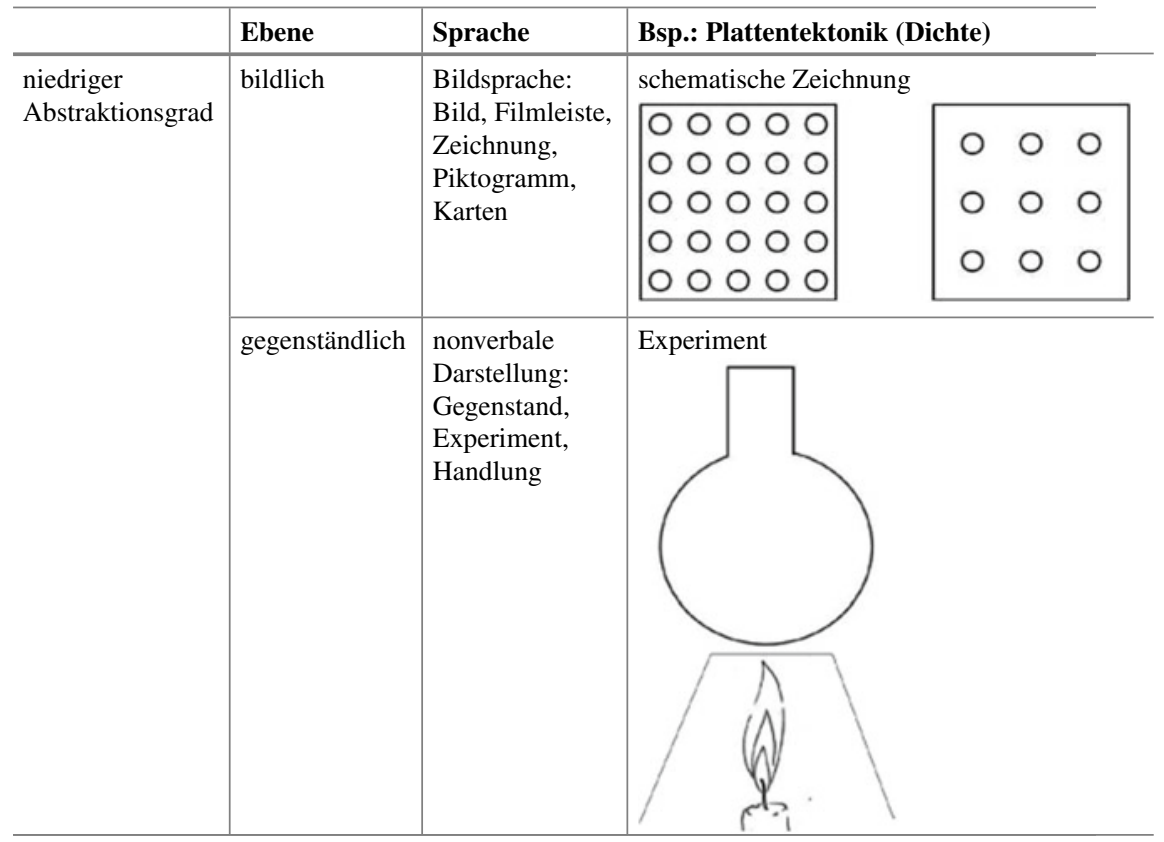

Quelle: verändert und erweitert nach Leisen 2013, 34, 36

\subsubsection{Empirische Befunde und konzeptionelle Arbeiten zu Sprache im Geographieunterricht}

In der geographiedidaktischen Forschung lassen sich nach Budke/Kuckuck (2017a) fünf interdisziplinäre und übergeordnete Diskurse zum Thema Sprache im Geographieunterricht identifizieren: (1) Integration durch Sprachförderung, (2) Sprachförderung im bilingualen Sachfachunterricht Geographie, (3) Förderung von Sprach- und Kommunikationskompetenz, (4) linguistic turn und (5) Inklusion durch Sprachförderung. Nachfolgend werden Arbeiten aus allen Bereichen, abgesehen von (4) linguistic turn, zu dem es bisher kaum Forschung gibt, beleuchtet. Insgesamt ist zu konstatieren, dass bisher wenige empirische Befunde, insbesondere in Blick auf Wirksamkeitsuntersuchungen, vorliegen.

Speziell für das Fach Geographie liegen empirische Erkenntnisse zum Zusammenhang von Sprachkompetenz und Fachkompetenz nur in Form eines Vergleichs von IGLU und TIMSS aus dem Jahr 2011 vor. Beim Vergleich der in IGLU 
erhobenen Leseleistungen mit den geographischen Kompetenzen, erhoben über TIMSS, zeigen sich deutlich positive Zusammenhänge zwischen den Kompetenzen Lesen und Geographie $(r=.69)$ (vgl. Bos et al. 2012b, S. 280). Ahrenholz (2010) untersuchte eine Sachunterrichtsstunde in der dritten Klasse hinsichtlich der Versprachlichung fachlicher Inhalte. Es zeigten sich bei Schüler*innen mit Migrationshintergrund große Schwierigkeiten. Doch auch einsprachig deutsche Kinder hatten Probleme bei der Verwendung neuer bildungssprachlicher Begriffe und arbeiteten immer wieder mit alltagssprachlichen Satzkonstruktionen (vgl. Ahrenholz 2010, S. 32). Losgelöst vom Geographie- und Sachunterricht zeigt die Studie von Rank et al. (2018) bei der Untersuchung von 222 Vorschulkindern insofern analoge Befunde, als in dieser Studie die Kinder mit Deutsch als Zweitsprache ein ähnliches bildungssprachliches Niveau erreichen wie diejenigen mit Deutsch als Erstsprache. „Andererseits bestätigt sich der Befund, dass schon im Vorschulalter der Bildungshintergrund im Elternhaus und die kognitiven Fähigkeiten der Kinder signifikante Einflussgrößen auf die Erlangung (bildungs)sprachlicher Fähigkeiten sind" (Rank et al. 2018, S. 126). Weitere empirische Studien zum Zusammenhang, insbesondere von Fachsprache und Fachwissen stehen aus.

Im geographieunterrichtlichen Kontext von Sprache und Fach ist der Bereich des Argumentierens bisher empirisch am intensivsten beforscht (vgl. Budke 2011; Budke/Creyaufmüller et al. 2015; Budke/Kuckuck et al. 2015; Budke/Uhlenwinkel 2013; Budke/Weiss 2014; Uhlenwinkel 2015). Argumentieren wird fächerübergreifend als ein wichtiger Weg zur Problemlösung benannt und für den Geographieunterricht als zentrale Bedingung für Lernerfolg herausgestellt (Budke et al. 2010, S. 182; vgl. Budke/Meyer 2015, S. 15). Insbesondere für multimodale, komplexe Problemstellungen kann das Argumentieren Schlüssel zum Verständnis sein (vgl. Leder 2015, S. 148). Auch bei der Argumentationskompetenz sind die drei oben genannten Rollen von Sprache Interaktion, Produktion und Rezeption bedeutsam. Das bedeutet, auf der einen Seite müssen Schüler*innen Argumentationen erkennen und in ihren einzelnen Bestandteilen verstehen können, auf der anderen Seite müssen sie eigene Argumentationen führen und mit ihnen interagieren können. Hierzu können sie sich Muster von Argumentationen bedienen (vgl. Budke et al. 2010, S. 183). Rezeptives und produktives Argumentieren kann auf unterschiedlichen Kompetenzniveaus stattfinden. Budke et al. (2010, S. 185) differenzieren vier Kompetenzstufen. Sie fordern Folgendes auf dem höchsten Niveau:

Schüler äußern zu einem geographischen Sachverhalt ihre Meinung und können diese mit überwiegend geeigneten, relevanten und gültigen Argumentationen begründen. 
Sie berücksichtigen den Adressaten ihrer Argumentation in angemessener Weise. Ihre Argumentation ist komplex und berücksichtigt mehrere Perspektiven.

Schüler erkennen, dass eine begründete Meinung zu einem geographischen Problem geäußert wird. Die Güte komplexer Argumente können sie anhand der Kriterien Relevanz, Eignung, Gültigkeit und Adressatenbezug richtig beurteilen. (Budke et al. 2010, S. 186)

Diese Kompetenzen werden im Geographieunterricht aber häufig nicht angesprochen. Insbesondere auf höheren Argumentationsebenen, etwa bei der Reflexion über Argumente, gibt es deutliche Lücken (vgl. Kuckuck 2015, S. 86). Unterrichtsbeobachtungen im Fach Geographie zeigen, dass in nur 8,4 \% der beobachteten Stunden Argumentation stattfindet (vgl. Budke 2012, S. 26). Besonders selten findet Argumentation in der Sekundarstufe I statt (5,5\% der Stunden im Vergleich zu 12,2 \% in der Sek. II) (vgl. Budke 2012, S. 26). Argumentationskompetenz kommt also anscheinend insgesamt eine geringe Bedeutung im Schulalltag zu (vgl. Kuckuck 2014, S. 101). Schulbuchanalysen der Bundesländer Berlin, Brandenburg und Nordrhein-Westfalen ergaben, dass nur 6,7 \% der Aufgaben Argumentationen fördern, wobei ein Großteil dieser Aufgaben auf die mündliche Argumentation in den Bereichen Produktion und Interaktion fällt (vgl. Budke 2011, S. 257-259). Die empirischen Untersuchungen im Bereich Argumentieren legen nicht nur offen, dass das Einüben von Argumentieren im Geographieunterricht eine marginale Rolle einnimmt, sondern auch, dass Schüler*innen gezielt Unterstützung zur Erlangung von Argumentationskompetenzen benötigen (vgl. Uhlenwinkel 2015, S. 59) und die Argumentationskompetenz bei Abiturient*innen nicht ausreichend ist, wie die Analyse von 60 Abiturklausuren im Fach Geographie in NRW zeigt (vgl. Budke/Weiss 2014).

Ein weiterer ausführlicher untersuchter Forschungsbereich von Sprache im Geographieunterricht, insbesondere in Form von konzeptionellen Arbeiten, ist bilingualer Unterricht (vgl. Böing 2009; Klein 2017; Lenz 2013; Meyer et al. 2017; Morawski 2019; Serwene 2017). Die Besonderheit bilingualen Sachfachunterrichts ist, dass Inhalt und Sprache zusammen gedacht werden; Sprache erreicht dadurch einen anderen Stellenwert als im herkömmlichen Geographieunterricht. Vor allem im Kontext der genannten Erkenntnisse zur Bedeutsamkeit vom Einbezug der Erstsprache im Fachunterricht bereichert bilingualer Unterricht den Diskurs, denn sobald Sprache (auch) die Funktion einer Arbeitssprache hat, wird die Erstsprache der Lernenden nicht ausgeschlossen und kann im wörtlichen Sinne des Konzeptes ebenfalls im Unterricht Verwendung finden (vgl. 
Böing/Palmen 2013, S. 46). Eine Studie von Morawski/Budke (2017) zeigt positive Auswirkungen bilingualen Geographieunterrichts auf das Bewusstsein von Sprache im Fachunterricht:

The results show that bilingual geography teachers assume key values related to language-awareness in geographic language. These results strongly allow discussion of language-aware implications, particularly in terms of structuring, visualization and transparency of discourse functions language awareness. (Morawski/Budke 2017, S. 61)

Im Kontext von Sprache und Geographieunterricht ist neben den Schulbuchanalysen zu Argumentationsaufgaben auch eine Studie von Relevanz, die die Textschwierigkeit von Geographieschulbüchern von Klasse 5 bis 10 untersucht. Die Studie von Berendes et al. (2018) zeigte nach der Analyse von 2928 Texten nur teilweise Anhaltspunkte für eine systematische Zunahme der Textkomplexität im Hinblick auf Klassenstufen und Schulformen. Darüber hinaus fielen deutliche Unterschiede zwischen den Verlagen auf (vgl. Berendes et al. 2018, S. 525). Diese Inkonsistenz hinsichtlich der Textschwierigkeit stützt die Annahme, dass unklar ist, was einen Schulbuchtext sprachlich und fachlich angemessen für eine bestimmte Niveaustufe macht - oder aber, dass zu wenig über die Angemessenheit von Schulbuchtexten reflektiert wird. Dass ein angemessener Schulbuchtext so geschrieben ist, dass er die Schüler*innen zwischen dem aktuellem und nächsten Kompetenzniveau abholt (vgl. Berendes et al. 2018, S. 519), ist angesichts der hohen Heterogenität innerhalb der Klassen ein schwammiger und daher gleichfalls schwierig zu operationalisierender Anspruch. Nichtsdestotrotz bestehen Versuche, Texte angemessen zu kategorisieren. So analysiert das Regensburger Analysetool für Texte Ratte Texte nach Kriterien wie Umfang, Syntax und Wortlänge und ordnet sie einer Jahrgangs- und Schwierigkeitsstufe zu (vgl. Wild/Pissarek o. J.). Vor dem Hintergrund angemessener Textschwierigkeit und dem entsprechenden Textverständnis sind die Ergebnisse einer Studie von Härtig et al. (2019) bemerkenswert. In drei experimentellen Studien mit randomisierten Gruppenvergleichen wurde das Textverständnis aus verschieden schwierigen Texten desselben Inhalts in einem Prä-Post-Design verglichen. Es zeigte sich, dass die Schüler*innen in allen Textvarianten gleich viel lernten (vgl. Härtig et al. 2019, S. 281-284). Für die Verleger*innen und Autor*innen von Schulbüchern sowie für die Lehrpersonen und die vorliegende Studie könnte sich aus diesen Erkenntnissen auf der einen Seite der Anspruch ableiten, Texte im Geographieunterricht auf Lesbarkeitsmerkmale hin zu untersuchen sowie die ihnen angebotenen Lernmaterialien sorgfältig zu prüfen. Auf der anderen Seite 
bedeutet es auch, insbesondere nach dem Lesen zu prüfen, inwiefern das Gelesene verstanden wurde und gegebenenfalls mit weiteren Zugängen (z. B. einem Experiment, bildlicher Darstellung etc.; Stichwort Darstellungsvernetzung) das Verständnis sicherzustellen.

Im internationalen Forschungskontext sind in Bezug auf Sprache und Geographieunterricht die weiter oben in diesem Kapitel zitierten Schulleistungsstudien TIMSS und IGLU zu nennen, die eine hohe Korrelation zwischen Geographieleistung und Leseleistung zeigen $(\mathrm{r}=.69)$. Weitere internationale Forschung, die sich explizit mit Fachsprache im Geographieunterricht beschäftigt, ist mir abgesehen von Erkenntnissen aus dem Bereich des bilingualen Geographieunterrichts nicht bekannt. Ein möglicher Grund hierfür könnte sein, dass fachsprachliche Anforderungen im Deutschen andere sind als beispielsweise im Englischen. So spielt für einen kompetenten Umgang mit Fachsprache im Englischen vor allem die lexikalische Ebene eine zentrale Rolle, während sich Aspekte der Syntax weniger stark von Alltagssprache unterscheiden.

Neben den dargestellten empirischen Erkenntnissen sind für die Konzeption und somit für die Design-Kriterien von sprachsensiblem Geographieunterricht ebenfalls bereits entwickelte Unterrichtsmaterialien für den Geographieunterricht mit sprachsensibler Ausrichtung von Belang (vgl. Budke/Morawski 2019; Czapek 2014; Düppe 2013; Felzmann/Conrad 2017; Heuzeroth 2017; Kniffka/Neuer 2008; Kuckuck/Röder 2017; Lindau/Renner 2017; Morawski et al. 2017a, 2017b; Oleschko et al. 2016; Recknagel/Hempowicz 2017; Reisch 2017; Reumont/Morawski 2017; Wassong/Kuckuck 2017; Weißenburg 2013). Besondere Berücksichtigung finden die Arbeiten von Conrad (2014, 2016) sowie Felzmann/Conrad (2017) in der Umsetzung der sprachsensiblen Geographieunterrichtsreihe, da diese thematisch besonders passend sind.

Sprache ist ausgemachtes Lernziel in den Bildungsstandards und nimmt unterschiedliche Rollen und Funktionen im Geographieunterricht ein, die explizit thematisiert werden müssen, um der Forderung nach Sprachsensibilität Nachdruck zu verleihen. Bisher gibt es verschiedene unvermittelt koexistierende konzeptionelle Ansätze und auch teilweise empirische Befunde, die auf die Notwendigkeit sprachsensibler Adressierung im Geographieunterricht hindeuten. Einen zentralen Schlüssel zum besseren Verstehen im Fach könnte die Vernetzung diskontinuierlicher und kontinuierlicher Darstellungsformen darstellen. Design-Kriterien, die als Grundlage für die Konzeption von sprachsensiblem Geographieunterricht herangezogen 
werden und die Grundlage für Wirksamkeitsuntersuchungen sein könnten, gibt es bisher allerdings nicht. Die aufgeführten geographiedidaktischen Erkenntnisse fließen in die Konzeption der Design-Kriterien ein und sind in dieser Form auch Bestandteil der vorliegenden Wirksamkeitsstudie.

\subsection{Theoretische und empirische Erkenntnisse zu Sprache im Fachunterricht aus anderen Fächern}

Insgesamt sind trotz zunehmender Forschungsarbeiten im Bereich Sprache und Fachunterricht bisher noch wenige evidenzbasierte Forschungsbefunde vorhanden. Während der Zusammenhang zwischen Fachsprache und Fachkompetenz in vielen Fächern, allen voran der Mathematik, empirisch gut abgesichert ist, differenziert sich das Forschungsfeld im Bereich sprachsensibler Interventionen gerade erst aus. Im folgenden Abschnitt löse ich den Blick von der Geographiedidaktik und weite ihn auf Forschung in anderen Fächern. Denn für das Fach Geographie als Brückenfach zwischen Gesellschafts- und Naturwissenschaft liegt es nahe, dass gleichfalls Erkenntnisse aus den benachbarten Fächern nutzbar gemacht werden können. Besonders weit fortgeschritten ist in diesem Feld die Forschung in der Mathematikdidaktik.

\subsubsection{Fach- und Sprachkompetenz im Mathematikunterricht}

Der Zusammenhang zwischen Sprachkompetenz und Mathematikleistung ist empirisch zwischenzeitlich vielfach belegt (vgl. Prediger 2019, S. 20). Beim Vergleich der Ergebnisse von IGLU und TIMSS zeigen sich deutlich positive Zusammenhänge zwischen den Kompetenzen in Lesen und Mathematik ( $\mathrm{r}=.54$ ). 2011 fiel der Erhebungszeitpunkt beider Studien zusammen, sodass die Studien mit gemeinsamer Stichprobe von 3928 Schüler*innen durchgeführt werden konnten (vgl. Bos et al. 2012a, S. 237). In einer Längsschnittstudie untersuchten Paetsch et al. (2016) bei 3169 Schüler*innen von der vierten bis zur sechsten Klasse den Einfluss der Lesekompetenz auf die mathematische Konzeptentwicklung. Es zeigte sich, dass auch unter Kontrolle des sozioökonomischen Status und der allgemeinen kognitiven Grundfähigkeiten ein signifikanter Zusammenhang besteht. Im Längsschnitt zeigte sich außerdem, dass die Lesekompetenz den mathematischen Lernzuwachs teilweise vorhersagen kann (vgl. 
Paetsch et al. 2016, S. 37). Ebenfalls einen Zusammenhang zwischen Rechenund Schriftsprachkompetenz untersuchten Schwenck/Schneider (2003) bei 115 Erstklässler*innen. Mittels einer qualitativen Clusteranalyse identifizierten sie vier verschiedene Kompetenzgruppen hinsichtlich der Rechen- und Lesekompetenz. Durch Mittelwerts- und Einzelvergleiche sowie einer Diskriminanzanalyse mit anschließender Reklassifikation ließen sich Hinweise darauf finden, dass kombinierte Schwächen in Rechen- und Leseleistung häufiger vorkommen als bis dato angenommen (vgl. Schwenck/Schneider 2003, S. 264). In einer qualitativen Untersuchung von Kern et al. (2017) mit förderbedürften Grundschulkindern stellten sich fachsprachliche Strukturen ebenfalls als ein erschwerender Faktor beim Erwerb von Rechenstrategien heraus (vgl. Kern et al. 2017, S. 241-242). Während sich die bislang angeführten Studien auf den Primarbereich beziehen, zeigt die Forschung von Prediger et al. (2015) mit 1495 Zehntklässler*innen ebenfalls Evidenz für den Sekundarbereich. In der Untersuchung ergaben sich hohe Korrelationen zwischen der Sprachkompetenz und der Mathematikleistung bei den Zentralen Prüfungen in der 10. Klasse (vgl. Prediger et al. 2015, S. 2324). Eine weitere Studie von Heppt et al. (2016) mit Viertklässler*innen (N $=22.015)$ stellt dar, dass fachsprachliche Kompetenz höher mit den erreichten mathematischen Leistungen korreliert als die alltagssprachliche Kompetenz (vgl. Heppt et al. 2016, S. 248-249).

Diverse Studien, die den Zusammenhang zwischen Sprachkompetenz und Fachkompetenz im Fach Mathematik untersucht haben, fokussieren (auch) auf die Herkunft und Erstsprache der Schüler*innen. So zeigt die quantitative Studie von Heinze et al. (2007) mit 556 Erstklässler*innen auf, dass Kindern mit Migrationshintergrund bei Kontrolle der kognitiven Grundfähigkeiten in manchen Bereichen des mathematischen Wissensaufbaus benachteiligt sind. Diese Unterschiede verschwinden, wenn der alltagssprachliche Sprachstand kontrolliert wird. Heinze et al. (2007) interpretieren dies als Hinweis auf den Einfluss der Deutschkompetenz beim Aufbau mentaler Repräsentationen oder allgemeiner auf den Erwerb mathematischer Kenntnisse im Deutschen. Eine Längsschnittstudie von Heinze et al. (2011) mit 292 Schüler*innen über einen Zeitraum von drei Jahren ergab, dass die sprachliche Kompetenz in Deutsch zu Beginn der Grundschulzeit für die Mathematikkompetenz zum Ende der Klassenstufe 1, 2 und 3 relevant ist. Disparitäten zwischen Kindern mit und ohne Zuwanderungshintergrund lagen bei bis zu einem Drittel der Standardabweichung in der Mathematikkompetenz. Bei Kontrolle der bei Schuleintritt diagnostizierten Sprachkompetenz im Deutschen kam es zu keinem Zeitpunkt mehr zu signifikanten Unterschieden. Dass die Sprachfähigkeit in der Zweitsprache Deutsch zu Beginn der Grundschulzeit prädikativ für die Mathematikkompetenz in der 
vierten Jahrgangsstufe unter Kontrolle der allgemeinen kognitiven Grundfähigkeiten ist, zeigte auch die Datenauswertung von 502 Viertklässer*innen (67 \% mit Migrationshintergrund) der Längsschnittstudie BeLesen (vgl. Mücke 2007, S. 279). Eine Studie von Paetsch et al. (2015) mit 370 Schüler*innen nichtdeutscher Herkunftssprache der dritten Klasse legt dar, dass Lesekompetenz und Wortschatzkenntnisse deutlich mit der Mathematikkompetenz zusammenhängen. Dieser Zusammenhang wurde nicht nur bei sprachlich anspruchsvollen Aufgaben, sondern auch bei einfacheren Aufgaben deutlich (vgl. Paetsch et al. 2015, S. 25-26). Ferner stellt Bernardo (2005) in Untersuchungen mit 111 bilingualen philippinisch-englischen Viertklässler*innen höhere Lösungshäufigkeiten bei mathematischen Textaufgaben fest, die in der Erstsprache der Kinder gestellt werden (vgl. Bernardo 2005, S. 420-421). Dieser Befund deutet auf einen Einfluss der Sprachkompetenz auf die mathematische Kompetenz von Schüler*innen und möglicherweise auf die Notwendigkeit des Einbezugs der Erstsprache im Mathematikunterricht hin. Eine Studie von Rehbein (2011), bei der türkischsprachige Viertklässer*innen in Gruppenarbeit während der Bearbeitung mathematischer Aufgaben ohne Lehrkräfte aufgenommen wurden, unterstützt die Forderung nach Einbezug der Erstsprache in den Mathematikunterricht ebenfalls. Es zeigte sich in der Studie, dass die Kinder zur mathematischen Aufgabenbewältigung auch Türkisch miteinander sprechen und nicht wie erwartet nur in der Schulsprache kommunizieren. Rehbein schlussfolgert daraus, dass die Erstsprache der Kinder mehr Akzeptanz als Arbeitssprache im Unterricht finden muss und spricht sich für eine Abkehr von dogmatischer Einsprachigkeit an Schulen aus (vg. Rehbein 2011, S. 227-228). Auch Clarkson (2006) beobachtet, dass Schüler*innen zwischen Erst- und Zweitsprache beim Mathematiklernen wechseln; er untersuchte 85 vietnamesisch sprechende Australier*innen in der vierten Klasse (vgl. Clarkson 2006, S. 195-196). Zu ähnlichen Ergebnissen kommt eine Laborstudie mit 31 Schüler*innen der Klassen 4-6 mit türkischem Migrationshintergrund. In halboffenen Interviews wurde untersucht, in welcher Sprache die Schüler*innen die Aufgabe eher lösen. Es zeigte sich hier, dass der Einbezug der Erstsprache Türkisch teils positive Effekte auf die Lösung der Aufgaben hatte. Die Autor*innen empfehlen daher, die Erstsprache der Schüler*innen möglichst in den Unterricht miteinzubeziehen und nicht per se auszuschließen (vgl. Meyer/Prediger 2011, S. 201).

Obwohl die Forschungslage in der Mathematikdidaktik zur Fachsprache fortgeschritten ist, liegt ein weiteres Desiderat in der Erörterung dessen, was genau erschwerende sprachliche Faktoren (auch in anderen Fächern) sind (vgl. Prediger 2019, S. 23). Gogolin et al. (2004) untersuchen 136 Siebtklässler*innen von insgesamt drei Gymnasien und drei Hauptschulen hinsichtlich ihrer sprachlichen 
Kompetenz in Deutsch und deren Bedeutung für das Mathematiklernen. Insbesondere komplexe Satzstrukturen und weitere grammatische Besonderheiten werden als erschwerender Faktor beim Mathematiklernen betrachtet; dem nachgeordnet sind Elemente auf lexikalischer Ebene.

In Bezug auf spezifische mathematikorientierte sprachliche Fähigkeiten ist evident, dass eine hinreichende Anzahl präzise gebrauchter grammatischer Einheiten als Steuerungselemente notwendig sind, um sachdienlich und zielgerichtet vorzugehen. Weniger bedeutend scheint hingegen das Verfügen über lexikalische Einheiten, eingeschlossen den sog. fachlichen Wortschatz. (Gogolin et al. 2004, S. 151)

Fach- und Bildungssprache werden auch in den Untersuchungen zum Argumentieren im Fach Mathematik von Prediger/Hein (2017) durchgehend als erschwerende Faktoren ermittelt. In der qualitativen Design-Studie ( $=20 ; 14-$ bis 15-jährige Schüler*innen) wurden die mündlichen und schriftlichen Versuche untersucht, logische Zusammenhänge beim mathematischen Beweisen im Bruchrechnen auszudrücken. Es zeigten sich erste Einsichten in die Komplexität der syntaktischen und lexikalischen Anforderungen im Zusammenhang mit logischen Verbindungen. Frank/Gürsoy (2015) stellen in einer Übersicht verschiedener Forschungsprojekte ebenfalls heraus, dass die sprachlichen Anforderungen im Mathematikunterricht aufgrund der spezifischen Textsorten hoch sind. So seien für das erfolgreiche Lösen von Textaufgaben ein fachsprachliches Repertoire, Strategien zum Umgang mit Sprache sowie Wissen über die Aufgaben und Kennzeichen der Textsorten notwendig (vgl. Frank/Gürsoy 2015, S. 155). Die sprachlichen Anforderungen im Mathematikunterricht werden in verschiedenen weiteren Beiträgen gesammelt (Clarkson 2009; Duval 2006; Gellert 2011; Rösch/Paetsch 2011); ein Konsens darüber, was genau Elemente von mathematischer Fachsprache sind, besteht allerdings noch nicht. Dass in den Fachdidaktiken noch nicht ausreichend Klarheit darüber besteht, welche sprachlichen Elemente für den Fachunterricht zu fokussieren sind, zeigt auch das Ergebnis einer quantitativen Umfrage unter 345 Lehrpersonen sowie sogenannten Sprachfördercoaches, Personen für Lehrer*innenfortbildungen zum Thema Sprachförderung, von Jost et al. (2017). Demgemäß wird die Unterscheidung Alltagssprache und Fachsprache zwar von Mathematiklehrkräften als relevant identifiziert, Sprachfördercoaches hingegen benennen ein viel differenzierteres Spektrum an sprachlichen Phänomenen als Grundlage für erfolgreiche Lernprozesse im Fachunterricht. 
$\mathrm{Zu}$ der Frage, wie sprachsensibler Mathematikunterricht aussehen soll, gibt es unterschiedliche Ansätze, die in ihrer Methodik oft der DaZ- und Fremdsprachendidaktik entlehnt sind (vgl. Prediger 2019, S. 23). Dabei wird es als zentral erachtet, dass Fachsprache nicht isoliert gelernt wird, sondern immer im fachlichen Kontext authentisch eingebettet ist (vgl. Linnemann et al. 2017, S. 282). Sprachliches Scaffolding ist in diesem Kontext in vielen empirischen Arbeiten aus der Mathematikdidaktik gewinnbringend. Beim Scaffolding handelt es sich um einen konstruktivistischen Lehr-Lern-Ansatz, in dem die Lernenden durch die Bereitstellung gestufter Hilfen beim Lernprozess unterstützt werden (für nähere Ausführungen vgl. Abschn. 3.3). Prediger/Wessel (2013) untersuchten ein sprachsensibles Treatment zu Brüchen. Dabei zeigten sich ebenfalls positive Effekte des sprachlichen Scaffoldings in der Experimentalgruppe (vgl. Prediger/Wessel 2013, S. 448). Eine weitere Erkenntnis war, dass das sprachliche Scaffolding in der Unterrichtskommunikation für die Lehrkräfte - trotz vorangehender Schulung - herausfordernd ist und die Qualität der Umsetzung stark variiert (vgl. Prediger/Wessel 2013, S. 454). Umfassend beschäftigte sich Wessel (2015) in ihrer Dissertation mit den Effekten sprachsensiblen Mathematikunterrichts auf die fachlichen Mathematikkompetenzen im Themenkomplex Brüche unter Einbezug von sprachlichem Scaffolding und Darstellungsvernetzung. Sie untersuchte mit dem Design-Based Research-Ansatz 303 Schüler*innen der Sekundarstufe I und stellte Effekte in Hinblick auf einen positiven fachlichen Kompetenzzuwachs fest. Zwischenzeitlich ist die Forschung soweit gediehen, dass zwei Interventionsansätze beim Lernen mit Brüchen gegenübergestellt werden können und die verschiedenen Sprachhintergründe der Schüler*innen Berücksichtigung finden (ein- vs. mehrsprachig). Auch an dieser Stelle zeigen sich für beide Interventionsformen im Vergleich zur Kontrollgruppe Vorteile, wobei keine signifikanten Unterschiede zwischen den Interventionen ausgemacht werden konnten (vgl. Prediger/Wessel 2018, 12-13). Die Forschung von Dröse/Prediger (2019) geht auf Basis aktueller Empirie davon aus, dass es bei der Bewältigung von Textaufgaben facheigene Strategien braucht. Sie entwickeln daher Design-Kriterien und ein entsprechendes Treatment, das zentral auf Scaffolding basiert (vgl. Dröse/Prediger 2019, S. 112-116). Kernerkenntnis ihrer Forschung ist, dass fachspezifische Lesestrategien für das fachliche Verständnis notwendig und hilfreich sind. Dröse (2019) beschäftigt sich in ihrer Dissertation mit dem Lesen und Verstehen von Textaufgaben im Mathematikunterricht und entwickelt dabei Design-Kriterien für eine entsprechende Unterrichtsreihe, die begleitend beforscht wird. Dabei spielt sprachliches Scaffolding ebenfalls eine zentrale Rolle. Die Begleitforschung gibt erste Hinweise auf die Wirksamkeit der sprachsensiblen Design-Kriterien für das Leseverständnis von Textaufgaben (vgl. Dröse 
2019, S. 300). Prediger/Zindel (2017) erreichten durch systematische Scaffolds beim Argumentieren ebenfalls positive Effekte auf Sprach- und Fachkompetenz bei den 94 untersuchten Schüler*innen der 8. bis 11. Klassen. Götze (2018) untersuchte die schriftliche Erklärkompetenz operativer Zusammenhänge von Kindern im Mathematikunterricht und zeigte, dass sprachlich-lexikalische Unterstützung in Form von Scaffolds die Erklärkompetenz verbessert. Prediger (2019, S. 23) fasst vier Kriterien für sprachsensiblen Matheunterricht zusammen, die sich bisher in der Mathematikdidaktik empirisch bewährt haben: (1) konsequente Diskursanregung, (2) Darstellungsvernetzung, (3) Scaffolding, (4) Vielzahl kommunikationsaktivierender Methoden und sprachlicher Unterstützungsformate.

Aus vielen der genannten Studien ist empirisch geprüftes Unterrichtsmaterial entstanden, das für die vorliegende Arbeit gesichtet und, soweit möglich und sinnvoll, in der eigens entwickelten Unterrichtsreihe berücksichtigt wurde. Weitere konzeptionelle Arbeiten sind ebenfalls von Relevanz, auch wenn diese nicht in empirische Wirksamkeitsforschung eingebunden waren (vgl. Wellenreuther 2010). Umfassendes Material gibt es auch auf der Website des Deutschen Zentrums für Lehrerbildung Mathematik (DZLM o. J.) sowie aus Projekten auf der Webseite der Universität Dortmund (Technische Universität Dortmund o. J.). Weitere Studien und einen guten Überblick über diese stellen Meyer/Tiedemann (2017, S. 47-58) sowie Duarte et al. (2011, S. 39-44) dar.

\subsubsection{Fach- und Sprachkompetenz in den naturwissenschaftlichen Fächern}

Beim Vergleich der IGLU- und TIMSS-Studien zeigt sich für die Naturwissenschaften ein hoher Zusammenhang zwischen Sprache und Fachleistungen ( $\mathrm{r}=$ .74) (vgl. Bos et al. 2012a, S. 237). Empirische Forschung im Fach Physik deutet ebenfalls darauf hin, dass Fachsprache ein eigener Kompetenzbereich im Fachunterricht ist. Härtig (2010) prüfte in einer explorativen Studie mit $\mathrm{N}=99$ den Zusammenhang zwischen der physikbezogenen Lesekompetenz, der allgemeinen Lesekompetenz sowie physikbezogenem Fachwissen. Die Ergebnisse deuten darauf hin, ,das Begriffsverständnis als Aspekt der Fachsprache als von Fachwissen und Unterrichtssprache unterscheidbare Fähigkeit aufzufassen“ (Härtig et al. 2012, S. 388). Höttecke et al. (2017) konnten feststellen, dass die fachsprachliche Kompetenz im Fach Physik - anders als in Sport - in der Notengebung der Lehrpersonen Berücksichtigung findet (vgl. Höttecke et al. 2017, S. 15). Weitere Untersuchungen in Chemie und Biologie deuten außerdem darauf hin, dass die Besonderheiten von Fachsprache das fachliche Verständnis erschweren. Deppner 
(1989) folgert in ihrer Studie im Chemieunterricht, dass unzureichende Sprachkompetenz das Lernen im Fach Chemie behindert. In ihren Untersuchungen gab ein Großteil der 275 Schüler*innen der 8. Klasse an, die fachlich angemessene Versprachlichung komplexer Inhalte schwierig zu finden (vgl. Deppner 1989, S. 195). Für das Fach Biologie erwiesen sich Testaufgaben mit Fachbegriffen als signifikant schwieriger als solche mit Alltagsbegriffen, was eine groß angelegte Studie von Schmiemann (2011) mit 3337 Schüler*innen aus den Klassen 5-10 von Haupt-, Real und Gesamtschulen sowie Gymnasien darlegt (vgl. Schmiemann 2011, S. 125-127). Auch in der Studie von Beerenwinkel/Gräsel (2005) teilen die Lehrkräfte diese Einschätzung in Bezug auf das Fach Chemie (vgl. Beerenwinkel/Gräsel 2005, S. 30). Theoretische Überlegungen und erste empirische Untersuchungen zu sprachlichen Anforderungen von Testaufgaben bestätigen den Befund von Schmiemann für weitere Fächer (Mathematik, Physik, Musik, Sport, Deutsch). Heine et al. (2018) modellierten für jedes Fach fünf Testaufgaben in je drei sprachlich unterschiedlich schwierigen Versionen. Es zeigte sich in der Pilotierung der Studie $(\mathrm{N}=601)$, dass die Lösungswahrscheinlichkeit von der einfachsten zur schwierigsten sprachlichen Version abnahm. Dies legt nahe, dass Sprache die Aufgabenschwierigkeit beeinflusst, obschon auch weitere Merkmale für die Aufgabenschwierigkeit maßgeblich sind (vgl. Heine et al. 2018, S. 89).

Es bestehen also vielfach Hinweise darauf, dass Fachsprache eine wichtige, erschwerende Rolle beim Erwerb von naturwissenschaftlicher Fachkompetenz spielt und demgemäß auch explizit im Fachunterricht adressiert werden sollte. Fachsprache in einem breiten und tiefen Verständnis ist bisher aber noch nicht empirisch untersucht. Studien fokussieren hingegen meist auf die lexikalische Ebene; allem voran Fachbegriffe. Ein umfassendes Verständnis von dem, was Fachsprache in den Naturwissenschaften ausmacht, zeigen Überlegungen zur Entwicklung eines Erhebungsinstruments im Fach Biologie. Dabei werden sowohl symbolische Aspekte als auch bildliche und verbale Repräsentationen in das Verständnis von Fachsprache integriert (vgl. Nitz et al. 2012, S. 121). Auch Schroeter-Bauss et al. (2018, S. 109-131) bestimmen eine Vielzahl fachsprachlicher Elemente. Insbesondere die Tatsache, dass viele physikalische Fachbegriffe auch in der Alltagssprache verwendet werden, sich dort aber jedenfalls teilweise von der fachlichen Bedeutung unterscheiden, erschwert das Begriffslernen (vgl. Tajmel 2010, S. 253). Bereits im naturwissenschaftlichen Anfangsunterricht in der Grundschule gibt es bildungssprachliche Diskursfunktionen (vgl. Hövelbrinks 2017, S. 199). Im Fach Physik wurden in den vergangenen 40 Jahren vermehrt Schulbuchanalysen hinsichtlich der Anzahl von Fachbegriffen durchgeführt. Der Befund bleibt über die Jahre hinweg mehr oder weniger konstant (vgl. Härtig 2010, S. 27): Eine Vielzahl an Fachbegriffen prägt Physikschulbücher. Anfang 
der 1980er-Jahre zählten Brämer/Clemens (1980, S. 77) in der Sekundarstufe I in jedem Physikschulbuch ca. 2000 Termini. $69 \%$ davon werden in nur einem von fünf Fachbüchern verwendet; die Hälfte aller Termini in einem Buch werden nur ein einziges Mal benutzt (Merzyn 1994). In aktuellen Untersuchungen zeigt sich, dass je nach Schulbuch in etwa 1000 Fachbegriffe genutzt werden, wobei pro Seite etwa zwei bis drei neue Begriffe eingeführt werden (vgl. Härtig/Kohnen 2017, S. 62). Zu ähnlichen Ergebnissen kam Fang (2006) bei Analysen von US-amerikanischen Schulbüchern der Middle School (11- bis 14-jährige Schüler*innen). Er stellt heraus, dass die Lesekompetenz ein bedeutender Faktor für das Verständnis naturwissenschaftlicher Schulbuchtexte ist, und betont die Notwendigkeit, Fachsprache als integralen und expliziten Bestandteil des Fachunterrichts zu behandeln (vgl. Fang 2006, S. 516). Auch im Physikunterricht wird die Forderung nach Sprachsensibilität gestellt. Fehlende Sprachsensibilität in Hinblick auf Mehrsprachigkeit sowie die Register Alltags-, Bildungs- und Fachsprache führe zu Intransparenz, ,wenn die Ziele von Unterrichtsstunden nicht mit sprachlichen Mitteln verdeutlicht werden und wenn es sprachlich nicht gelingt zu erklären, wie die einzelnen Phasen im Unterrichtsgeschehen zusammenhängen“ (vgl. Freckmann/Komorek 2019, S. 222). Ähnlich wie in der Mathematikdidaktik besteht auch in der Biologiedidaktik die Grundüberlegung, dass fachliche Lesekompetenz von der allgemeinen Lesekompetenz insoweit abweicht, als sich die Rolle von Fachwortschatz und bestimmten sprachlichen Strukturen von Fach zu Fach unterscheiden und Lesekompetenz daher speziell im Fachunterricht adressiert werden muss (vgl. Cromley et al. 2010, S. 694). Ebenfalls für den Biologieunterricht untersuchen Schmellentin et al. (2017) Schulbuchtexte auf ihre Verständlichkeit hin und identifizieren zentrale sprachliche Anforderungen darin. Sie kommen zu dem Ergebnis, dass Texte nicht per se durch Weglassen einzelner Charakteristika vereinfacht werden können und sollten, geben aber Hinweise darauf, wie Texte punktuell entlastet werden können und das Textverständnis verbessert werden kann (vgl. Schmellentin et al. 2017, S. 88). In einer weiteren Untersuchung von Schulbuchtexten für das Fach Biologie zeigt sich, dass die Texte zwar immer mit didaktischem Anspruch geschrieben werden, dies aber nicht unbedingt zu tatsächlich verständlichen Texten führt. In dem Forschungsprojekt wurden daher verschiedene Prinzipien erarbeitet, die als Hilfestellung für die Gestaltung von Schulbuchtexten herangezogen werden können (vgl. Schneider et al. 2019, S. 81). Auffällig ist, dass der Bedeutsamkeit von Bildungs- und Fachsprache in naturwissenschaftlichen Schulbuchtexten ihr tatsächlicher Einbezug im Unterricht jedenfalls teilweise entgegenzulaufen scheint. In einer Studie von Beerenwinkel/Gräsel (2005, S. 27) gaben $40 \%$ der 240 befragten Chemielehrkräfte 
an, höchstens zwei Mal pro Halbjahr Texte im Chemieunterricht der Sekundarstufe I einzubeziehen. Schulbuchtexte wurden von den Lehrpersonen außerdem als eher nicht interessant für die Schüler*innen sowie wenig zielführend eingestuft, was die Verbesserung eines themenübergreifenden Verständnisses angeht. Die Texte werden meistens zur Wiederholung eingesetzt, ein aktiver Umgang mit ihnen im Unterricht findet kaum statt (vgl. Beerenwinkel/Gräsel 2005, S. 30).

Bisher gibt es nur wenige Interventionsstudien zu sprachsensiblem naturwissenschaftlichem Unterricht. Die wenigen vorliegenden legen nahe, dass gezielte Sprachförderung im naturwissenschaftlichen Fachunterricht dem fachlichen und fachsprachlichen Verständnis förderlich ist. Agel et al. (2012) führten mit 24 mehrsprachigen Schüler*innen mit sehr geringer Sprachkompetenz eine Intervention zum Schreiben von Versuchsprotokollen unter Einbezug der Erstsprache durch. Am Ende der Intervention erreichten die Schüler*innen in dem geförderten Bereich „Protokolle schreiben“ den Klassendurchschnitt. Für das Fach Chemie entwickelten Schmölzer-Eibinger/Langer (2010) ein 3-Phasen-Modell zur Förderung von Textkompetenz. In der praktischen Erfahrung zeigten sich durch die drei Phasen „Wissensaktivierung“, „Arbeiten an Texten“ und „Texttransformation" positive Effekte auf die sprachlichen und inhaltlichen Lernaktivitäten (vgl. Schmölzer-Eibinger/Langer 2010, 216-217). Trotz der wenigen Interventionsstudien gibt es für den sprachsensiblen naturwissenschaftlichen Unterricht bereits zahlreiche konzeptionelle, teils durch empirische Forschung entwickelte und teils unterrichtspraktisch bewährte, Ansätze (vgl. Böttcher/Meister 2015; Heise/Höttecke 2006; Höttecke 2006; Leisen 2011; Leisen/Seyfarth 2006). Durch die Überlegungen zu Charakteristika von Fachsprache gibt es auch Ansätze für verschiedene Erhebungsinstrumente. Häufig eingesetzt werden der C-Test sowie die Profilanalyse, die ich im Rahmen der vorliegenden Arbeit ebenfalls einsetze (vgl. Nitz et al. 2012; Pineker-Fischer 2017; Schroeter-Bauss et al. 2018). Lumer/Winter (2019) entwickelten ein Lehr-Lern-Konzept zur Förderung von Sprach- und Leseverstehen im Biologieunterricht der Sekundarstufe II, das Leseverständnis und diskontinuierliche Darstellungsformen in sein Zentrum rückt und daher ebenfalls Berücksichtigung findet.

\subsubsection{Fach- und Sprachkompetenz in den Gesellschaftswissenschaften}

Auch in den gesellschaftswissenschaftlichen Fächern spielt Sprache eine zentrale Rolle; dies zeigt sich zuvorderst in ihrer Nennung in den Curricula (vgl. Handro 2018, S. 13). In den verschiedenen Fächern ist der sprachliche Aspekt aktuell 
noch unterschiedlich stark erforscht. Während im Fach Politik noch kaum empirische Forschung vorhanden ist (vgl. Oleschko 2015, S. 83), beschäftigt sich die Geschichte schon länger mit den Anforderungen von Sprache im Fach. Insgesamt ist die Forschungslage im Vergleich zu den naturwissenschaftlichen Fächern und Mathematik weniger fortgeschritten.

In Geschichte wird die Bedeutung von Sprache zentral hervorgehoben. Insbesondere die Funktion der Erkenntnisgewinnung durch Sprache steht im Zentrum. In der Forschung sind daher insbesondere narrative Kompetenzen und die dazu passenden Lernaufgaben und Operatoren im Blick (vgl. Handro 2018, S. 13). Dabei wird angenommen, dass der Geschichtslernprozess aus den eng miteinander verwobenen Komponenten Inhalt, Sprache und Denken besteht (vgl. Hartung 2018, S. 85). Das bedeutet auch, dass Operatoren nicht nur eine inhaltliche Ebene, sondern auch eine sprachliche Komponente haben. Besonders intensiv wurden begründe-Aufgaben empirisch untersucht; es zeigte sich in den Studien mehrfach, dass mit diesen Aufgaben hohe Anforderungen einhergehen, die nicht allein fachlich gerechtfertigt werden (Altun et al. 2015; vgl. Altun/Günther 2018). Ähnliche Anforderungen ergeben sich aus Operatoren im Politikunterricht (vgl. Oleschko 2015). Darüber hinaus stellen Begrifflichkeiten im Geschichtsunterricht Schwierigkeiten bzw. besondere Anforderungen dar. Denn historische Begriffe scheinen schwierig klassifizierbar und sie sind mehrfach mit verschiedenen realen Ereignissen belegt. So ist eine Revolution mit unterschiedlichen Kontexten verbunden, was das Verständnis aufgrund mangelnder Eindeutigkeit erschweren kann (vgl. Beilner 2002; Langer-Plän 2003; Langer-Plän/Beilner 2006; Sauer 2015). Probleme zeigen sich auch auf Ebene der Sprachproduktion hinsichtlich der Verwendung von Konnektoren. In einer Studie von Memminger (2009) offenbaren sich ,erhebliche Schwierigkeiten, wenn es darum geht, semantisch sinnvolle Verknüpfungen herzustellen. So ist eine z. B. finale oder kausale Verbindung der einzelnen historischen Fakten, die wirkliches historisches Erzählen ausmachen würde, relativ selten“ (Memminger 2009, S. 209).

Im Philosophieunterricht wird Sprache als das konstituierende Element betrachtet, das besondere Anforderungen an Lehrende und Lernende stellt. Brinkmeier (2019) nimmt an, dass vor allem auf lexikalischer Ebene Schwierigkeiten mit Polysemien und Metaphorik vorhanden sind.

Die Sprache ist der Philosophie wesentlich, und zwar insofern, als ihre eigentümlichen Erkenntnisgegenstände, die Begriffe, vermittels derer wir uns die Welt verständlich machen und von denen wir uns in unserem alltäglichen Tun und Treiben mehr oder weniger unbewusst leiten lassen, sprachlich konstituiert sind. Für den Philosophieunterricht folgt daraus unmittelbar, dass er in einem ganz bestimmten Sinn Sprachunterricht ist. (Brinkmeier 2019, S. 79) 
Wie sprachsensibler Philosophieunterricht aussieht, ist bisher nicht geklärt. Bei der Analyse einer Unterrichtsstunde im Fach Geschichte hat sich das Scaffolding (vgl. Abschn. 3.3) als anschlussfähig für das historische Begriffslernen erwiesen (vgl. Eichner et al. 2019, S. 258). In einer weiteren Untersuchung zeigten sich ebenfalls positive Effekte auf die historische Sinnbildung (vgl. Barricelli 2015, S. 45). Sprachsensibilität wird in der Geschichtsdidaktik auch im Sinne der Diversitätssensibilisierung verwendet, um ein reflektiertes Geschichtsbewusstsein zu fördern (vgl. Barricelli 2019, S. 30)

In den vergangenen zehn Jahren hat die Forschung um sprachsensiblen Fachunterricht in den Fachdidaktiken stark zugenommen. Dabei fokussieren viele Studien auf Kinder mit Deutsch als Zweitsprache sowie Kinder in der Grundschule. Erst allmählich mehren sich Studien zu sprachsensiblen Interventionen im Fachunterricht; der Zusammenhang zwischen Sprache und Fachleistung gilt hingegen in vielen Fächern als belegt. Bisherige Interventionsstudien nehmen insbesondere das sprachliche Scaffolding, also die Bereitstellung von gestuften Hilfen, in den Blick. Scaffolding zeigt sich in diesen Untersuchungen als wirksam und ist daher auch im Rahmen der Design-Kriterien für sprachsensiblen Geographieunterricht relevant.

Open Access Dieses Kapitel wird unter der Creative Commons Namensnennung 4.0 International Lizenz (http://creativecommons.org/licenses/by/4.0/deed.de) veröffentlicht, welche die Nutzung, Vervielfältigung, Bearbeitung, Verbreitung und Wiedergabe in jeglichem Medium und Format erlaubt, sofern Sie den/die ursprünglichen Autor(en) und die Quelle ordnungsgemäß nennen, einen Link zur Creative Commons Lizenz beifügen und angeben, ob Änderungen vorgenommen wurden.

Die in diesem Kapitel enthaltenen Bilder und sonstiges Drittmaterial unterliegen ebenfalls der genannten Creative Commons Lizenz, sofern sich aus der Abbildungslegende nichts anderes ergibt. Sofern das betreffende Material nicht unter der genannten Creative Commons Lizenz steht und die betreffende Handlung nicht nach gesetzlichen Vorschriften erlaubt ist, ist für die oben aufgeführten Weiterverwendungen des Materials die Einwilligung des jeweiligen Rechteinhabers einzuholen.

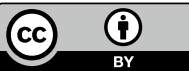

